\title{
Integrated data analysis at TJ-II: the density profile
}

B.Ph. van Milligen, ${ }^{1}$ T. Estrada, ${ }^{1}$ E. Ascasíbar, ${ }^{1}$ D. Tafalla, ${ }^{1}$ D. López-Bruna, ${ }^{1}$ A. López Fraguas, ${ }^{1}$ J.A. Jiménez, ${ }^{1}$ I. García-Cortés, ${ }^{1}$ A. Dinklage, ${ }^{2}$ and R. Fischer ${ }^{3}$

1) Asociación EURATOM-CIEMAT para Fusión, Avda. Complutense 22, 28040 Madrid, Spain

2) Max-Planck-Institut für Plasmaphysik, Teilinstitut Greifswald, EURATOM Association, Wendelsteinstr. 1, 17491 Greifswald, Germany

3) Max-Planck-Institut für Plasmaphysik, EURATOM Association, Boltzmannstr. 2, D-85748 Garching, Germany

(Dated: 7 June 2011)

An integrated data analysis system based on Bayesian inference has been developed for the TJ-II stellarator. It reconstructs the electron density profile at a single time point, using data from interferometry, reflectometry, Thomson Scattering, and the Helium beam, while providing a detailed error analysis. In this work, we present a novel analysis of the ambiguity inherent in profile reconstruction from reflectometry, and show how the Integrated Data Analysis approach elegantly resolves it. Several examples of the application of the technique are provided, in both low-density discharges with and without electrode biasing, and in high-density discharges with an (L-H) confinement transition.

PACS numbers: 07.05.Kf,52.25.Os,52.25.Vy,52.55.Hc 


\section{INTRODUCTION}

The goal of this work is to perform an integrated analysis of the data obtained using various diagnostics based on Bayesian inference ${ }^{1}$, applied to profile reconstruction at TJ-II.

TJ-II is a flexible Heliac $\left(R=1.5 \mathrm{~m}, a<0.2 \mathrm{~m}, B_{0}<1 \mathrm{~T}\right)$ with 4 periods and 32 toroidal field coils ${ }^{2}$. Heating is performed by the Electron Cyclotron Resonant Heating (ECRH) system (consisting of two gyrotrons and beam lines with an injected power of up to about $300 \mathrm{~kW}$ each); and Neutral Beam Injection (NBI), consisting of two injectors (co and counter with respect to the toroidal magnetic field $B_{\phi}$, with an injected power of up to about $500 \mathrm{~kW}$ port-through each).

TJ-II is an extremely well-diagnosed machine. Some of the available diagnostics are: interferometry, Thomson Scattering (TS), reflectometry, Helium beam, Lithium beam, Heavy Ion Beam Probe (HIBP), Electron Cyclotron Emission (ECE), Soft X-Ray Emission (SXR), etc. Up to now, no systematic attempt has been made to combine the information delivered by several of these diagnostics in order to maximize knowledge about plasma profiles. Evidently, such improved knowledge is important for, e.g., transport studies and the calculation of plasma performance parameters, with appropriate error bars.

In this initial work, we will limit ourselves to the analysis of density profiles $\left(n_{e}\right)$, leaving temperature profiles $\left(T_{e}\right)$, combined density and temperature profiles, effective charge $\left(Z_{\text {eff }}\right)$, electric potential $(\phi)$, etc., to future work. The temporal evolution of the profiles is not considered here; we limit ourselves to reconstructing an instantaneous profile at a specified time point. In Section II, we provide a general outline of the approach followed. In Section III. we describe how the various diagnostic systems are modeled and what assumptions

are made. In Section [V] we explain the structure of the program for reconstruction of the density profile, and how the error analysis is performed. In Section $\mathrm{V}$, we provide a few examples of profile reconstruction at TJ-II. Finally, in Section VI, we draw some conclusions concerning the method, its advantages and strengths, and discuss possible future extensions of this work. 


\section{INTEGRATED ANALYSIS OF PROFILE MEASUREMENTS}

The experimental documentation of fusion plasmas often requires a consistent analysis of data from different sources. For example, in this work we will discuss the reconstruction of the spatial profile of the electron density using the reflectometry, Thomson scattering, interferometry, and Helium beam diagnostics at TJ-II.

In the conventional approach, the combined analysis of profile measurements begins with the individual analyses of the concerned diagnostics. In a next step, the resulting data are transformed from laboratory coordinates to magnetic flux surface coordinates, based on equilibrium calculations. Finally, a model function is fitted to the analyzed data. Although conceptually straight-forward, this conventional approach suffers from deficiencies which may lead to unwanted ambiguities in the combined analysis. Examples of such issues arise from parametric entanglements, e.g., one of the diagnostic units could depend on the input from another measurement. Another example, although not of primary concern for the case discussed here, is the dependence of the aforementioned coordinate transformation on the plasma pressure - while the latter is an object of the measurement itself ${ }^{3}$. Moreover, beyond physical interdependencies, systematic effects affecting individual diagnostics, or $a$ priori inaccessible parameters such as offsets, further complicate the situation.

Bayesian analysis offers the possibility to integrate the analysis of heterogeneous measurement data and underlying physical models in a single framework, sometimes called Integrated Data Analysis (IDA) ${ }^{4}$. IDA has been applied to the combination of data from different experiments at Wendelstein 7 -AS $S^{516}$, ASDEX Upgrade (for a recent review cf. ${ }^{7}$ ), and JET ${ }^{8}$. In this framework, rather than analyzing all measurements separately and combining the results, a single model profile is used to fit all data simultaneously while explicitly formulating underlying assumptions, thus clarifying the limitations of the analysis (that would otherwise remain hidden). The approach is based on probability distributions, and allows a thorough and complete analysis of error propagation $\left.{ }^{9}\right)$. Bayes' theorem describes the formal essence of the implementation of IDA:

$$
P(\vec{\alpha} \mid \overrightarrow{\mathrm{d}}, \vec{\sigma}, I)=\frac{\mathcal{L}(\overrightarrow{\mathrm{d}} \mid \vec{\alpha}, \vec{\sigma}, I) \pi(\vec{\alpha} \mid I)}{\int \mathcal{L}(\overrightarrow{\mathrm{d}} \mid \vec{\alpha}, \vec{\sigma}, I) \pi(\vec{\alpha} \mid I) d \vec{\alpha}}
$$

The result $P$ is a probability distribution of the parameters $\vec{\alpha}$. We note that the parameter vector $\vec{\alpha}$ includes the parameters describing the density profile $\left(\vec{\alpha}_{n_{e}}\right)$ as well as other param- 
eters (e.g., diagnostic calibration factors), denoted by $\vec{\alpha}^{\prime}: \vec{\alpha}=\left\{\vec{\alpha}_{n_{e}}, \vec{\alpha}^{\prime}\right\} . P$ is a conditional probability distribution; it quantifies the probability to find a result $\vec{\alpha}$ given measured data $\overrightarrow{\mathrm{d}}$, the measurement uncertainties $\vec{\sigma}$ and context information $I$ summarizing all assumptions (e.g., the functional form of the fit function) entering the combined analysis. Eq. (1) tells us that the result of a measurement also depends on the knowledge (better: ignorance) of the parameters $\vec{\alpha}$ prior to the measurement, i.e., without data $\overrightarrow{\mathrm{d}}$ : this knowledge is encoded in the probability distribution $\pi(\vec{\alpha} \mid I)$. The data enter in the so-called likelihood $\mathcal{L}(\overrightarrow{\mathrm{d}} \mid \vec{\alpha}, \vec{\sigma}, I)$, specifying the probability of obtaining a measurement given the model parameters. The normalization of $P$ is not relevant for the problem of determining the most probable values and uncertainties of the parameters.

The key feature of the approach that allows 'fitting' a joint model for the density profile (encoded by $\vec{\alpha}_{n_{e}}$ ) to all data is now reflected by the explicit calculus of the likelihood: since $\mathcal{L}$ should measure the degree of compliance of the actual data with given parameters, it is necessary to determine the misfit of the modeled data $\vec{d}^{0}$ with regard to the measured data $\vec{d}$. In order to weight individual data points with respect to each other, the likelihood typically involves the measurement error of the data $\vec{\sigma}$. The modeled data $\overrightarrow{d^{0}}$ are determined by the forward function $\vec{f}$ which can be regarded as a deterministic model for a synthetic diagnostic

$$
\overrightarrow{d^{0}}=\vec{f}(\vec{\alpha})=\vec{f}\left(\vec{\alpha}_{n_{e}}, \vec{\alpha}^{\prime}\right)
$$

involving the (specific) diagnostic model parameters $\vec{\alpha}^{\prime}$.

To combine different measurements, each diagnostic must ultimately be represented by its own forward model. The crux of the integrated analysis is the use of the same physical model parameters $\left(\vec{\alpha}_{n_{e}}\right)$ for all measurements. This is the formal difference to the conventional approach for which individual diagnostics produce individual estimates of a physical quantity. It is this feature of the conventional approach which gives rise to practical difficulties, particularly when the data suffer from inconsistencies such as systematic shifts in the coordinate mapping.

Since the elaboration of the forward model forces one to formulate a model linking the physics underlying a measurement to the resulting observation, this forward model must necessarily contain all physics relevant to the measurement. E.g., in the case of interferometry, the model for the integration along the line of sight must include the transformation 
from an assumed density distribution (specified via $\vec{\alpha}_{n_{e}}$ ) on magnetic surfaces to laboratory coordinates. In other words, the formulation of the forward function (or the measurement model) is a way to consistently incorporate physical information into the analysis of data.

Regarding the integrated analysis of the electron density profile at TJ-II, note that the fact that the data from different diagnostics are taken independently implies that the likelihood can be factorized. Labelling each diagnostic by a subindex $j$, one has:

$$
\mathcal{L}(\overrightarrow{\mathrm{d}} \mid \vec{\alpha}, \vec{\sigma}, I)=\prod_{j} \mathcal{L}_{j}\left(\overrightarrow{\mathrm{d}}_{j} \mid \vec{\alpha}_{n_{e}}, \vec{\alpha}_{j}^{\prime}, \vec{\sigma}_{j}, I\right)
$$

The same applies to $\pi$ :

$$
\pi(\vec{\alpha} \mid I)=\pi\left(\vec{\alpha}_{n_{e}} \mid I\right) \times \Pi_{j} \pi_{j}\left(\vec{\alpha}_{j}^{\prime} \mid I\right)
$$

This formulation has a practical and highly relevant side-effect: Eqs. 3 and 4 allow a formal and quantifiable model for 'systematic errors': a parameter describing a systematic effect $\alpha_{s}^{\prime}$ can be introduced but its uncertainty must be quantified a priori by $\pi\left(\alpha_{s}^{\prime} \mid I\right)$. This allows assessing effects known to exist and to include their influence on the analysis result, even though their direct precise determination is impossible.

To find the best estimates and uncertainties of any parameter $\alpha_{i}$, one needs to determine the marginal probability distribution $P\left(\alpha_{i} \mid \overrightarrow{\mathrm{d}}, \vec{\sigma}, I\right)$ as a final step. The marginal distribution can be determined by the marginalization rule of probability theory:

$$
P\left(\alpha_{i} \mid \overrightarrow{\mathrm{d}}, \vec{\sigma}, I\right)=\int P(\vec{\alpha} \mid \overrightarrow{\mathrm{d}}, \vec{\sigma}, I) \mathrm{d} \alpha_{1} \cdots \mathrm{d} \alpha_{i-1} \mathrm{~d} \alpha_{i+1} \cdots \mathrm{d} \alpha_{n}
$$

This marginalization rule can be considered a generalized error propagation law, since the

marginalization propagates the uncertainties of the marginalized model parameters $\alpha_{j}, j \neq i$ to the marginal result.

\section{FORWARD MODELLING AND ERROR ESTIMATES}

In this Section, we will discuss various facts and assumptions underlying the modelling of the relevant measurements, and in particular their effect on the final error in the results. More details concerning the various measurement devices discussed can be found in the cited references. 


\section{A. Magnetic geometry}

The forward modelling of diagnostics in a magnetic confinement device requires knowledge about the magnetic geometry of the system. It is assumed that many relevant quantities (pressure, density, temperature) are constant on magnetic flux surfaces, due to the fact that transport along field lines is at least a magnitude faster than transport across. This assumption allows comparing (local) density measurements performed at different toroidal angles, in spite of the helical twist of the plasma, by translating the (local) measurements to a magnetic coordinate system.

At TJ-II, one typically relies on VMEC ${ }^{10}$ calculations at zero pressure to provide the magnetic geometry, or (equivalently) on a code that directly uses the Biot-Savart law to estimate the magnetic flux. The vacuum magnetic configuration is identified by a label consisting of three numbers proportional to the currents in the external coil sets. The assumed magnetic geometry is subject to the following error sources:

- Errors in the calculated vacuum magnetic field due to approximations used in the field source: the continuous current distribution in external coils is approximated by a finite number of filamentary currents. Earlier studies have determined the minimal number of filaments needed to achieve good convergence ${ }^{11}$. The resulting error is negligible.

- Errors due to model simplifications: magnetic islands are ignored.

- Errors due to the positioning of the external coils: the coil placement precision is at the mm level. Coil displacements could in principle produce error fields that might resonate with the main field, leading to the formation of (unwanted) islands. However, the experimental measurement of the vacuum field structure has shown that such error fields are insignificant ${ }^{12}$.

- Errors due to stray fields: TJ-II has been built using low-magnetization steel, and additional installations have also been analyzed carefully in this respect. Stray fields are expected to be insignificant.

- Errors in the amplitude of the currents in the external coils (measured to be below a few percent). Such errors would lead to a change of the effective magnetic configuration, mainly modifying the rotational transform and the radial position of the rational 
surfaces. The effect on the shape of the flux surfaces is, however, small, except when the modification of the rotational transform profile leads to the introduction of a loworder magnetic resonance (island) in the plasma.

- Errors due to net currents in the plasma. Net currents do sometimes flow inside the plasma, typically smaller than a few kA. These currents are associated with (a) the bootstrap current ${ }^{13}$, (b) an external Ohmic drive ${ }^{1415}$, (c) the drive by NBI (usually small), (d) the drive by ECRH (usually small except in current drive experiments) Such net currents (with a distribution that is often partly unknown) produce a modification of the magnetic field, thus modifying the configuration (mainly the rotational transform profile and the radial position of the rational surfaces).

- Errors due to finite pressure. In ECRH plasmas, $\langle\beta\rangle$ is small. In NBI plasmas, $\langle\beta\rangle$ may attain values of up to about $1 \%$. The induced Shafranov shift of the magnetic axis is approximately linear in $\langle\beta\rangle$ and is only about $3.5 \mathrm{~mm}$ for $\langle\beta\rangle=1 \%$. This small deformation of the magnetic geometry due to $\langle\beta\rangle$, even at the magnetic axis where this effect is largest, means that vacuum geometry generally provides a good approximation of the true geometry at finite pressure at TJ-II.

In summary, the magnetic geometry delivered by the vacuum calculations is expected to be quite close to the actual magnetic geometry except in the presence of significant islands. Therefore, we will limit our studies to plasmas without large islands and assume that the actual magnetic geometry is approximated satisfactorily by the vacuum magnetic geometry, as calculated.

Therefore, knowing the magnetic geometry and the location of the diagnostics in real space (also with good accuracy), it is possible to calculate the diagnostic response of some of these diagnostics to theoretical profiles (in the framework of forward modelling). We will assume that all relevant plasma quantities depend only on the toroidal magnetic flux, $\psi$ (i.e., the effective radius, $\rho=\sqrt{\psi_{N}}$, where the $N$ stands for "normalized": $\psi_{N}=\psi / \psi_{\mathrm{LCFS}}$, where $\psi_{\text {LCFS }}$ is the value of the flux at the Last Closed Flux Surface). In other words, assuming an input profile $n_{e}^{0}(\rho)$ one should be able to compute the relevant diagnostic responses without difficulty on the basis of the magnetic geometry, which provides a mapping from the cylindrical coordinates $(R, \phi, Z)$ to $\rho$. 
In principle, the flux surface mapping is only available within the LCFS. However, the density is not zero at the LCFS; it falls to zero within a few $\mathrm{cm}$ outside the LCFS. Since the density is parametrized in terms of $\rho$ (see next subsection), we will need to compute an approximate value of $\rho$ outside the LCFS, along the various diagnostic chords. To do so, we first compute $\rho$ along the chord $\rho\left(R_{\text {chord }}, Z_{\text {chord }}\right)$ inside the LCFS (at a constant value of $\phi$ ), and extrapolate linearly outside the LCFS. This extrapolation is reliable up to and slightly beyond $\rho=\rho_{\max }=1.2$.

\section{B. Parametrization of the density profile}

In the framework of modelling the diagnostic responses, one needs to express the model profile $n_{e}^{0}(\rho)$ via a parametric representation:

$$
n_{e}^{0}(\rho)=n_{e}^{0}\left(\rho, \alpha_{k}\right), k=1, \ldots, K,
$$

where the $\alpha_{k}$ are the profile parameters. In principle, one could use any simple polynomial expansion, or, e.g., splines $\frac{17}{17}$. We will use the Fourier-Bessel series, that can be written as $(\rho \geq 0)$ :

$$
n_{e}^{0}(\rho)=\sum_{k=1}^{K} \alpha_{k} J_{0}\left(\lambda_{k} \rho / \rho_{\max }\right),
$$

where $\lambda_{k}$ is the $k^{\text {th }}$ zero of the Bessel function $J_{0}$, and $\rho_{\max }=1.2$.

This expansion offers the following important advantages: (a) the expansion consists of functions with zero derivative at $\rho=0$ that drop to zero at $\rho=\rho_{\max }$. Therefore, the expansion will converge rapidly for profiles with the same properties, such as the density profile, which has zero gradient at $\rho=0$ since the particle flux, proportional to the gradient, must vanish there, while the profile must drop to zero at some point $\rho_{\max }>1$; (b) the expansion is complete (capable of approximating any continuous, square-integrable, function with arbitrary accuracy); (c) the expansion consists of orthogonal functions. Thus, the coefficients will be linearly independent, which serves to stabilize the maximization process discussed below. In fact, these functions satisfy the following orthogonality condition $(n, m \geqslant$ 1):

$$
\int_{0}^{1} J_{0}\left(\lambda_{n} \rho\right) J_{0}\left(\lambda_{m} \rho\right) \rho d \rho \propto \delta_{m n},
$$

which is an appropriate condition for radial basis functions in a cylindrical coordinate system. 


\section{Interferometry}

The microwave interferometer at TJ-II provides a value of the line-integrated electron density. The phase change of the interferometer beam as it traverses the plasma is computed by:

$$
\Delta \phi=\int(\eta-1) \frac{\omega}{c} d l
$$

where $\eta$ is the refractive index $\sqrt{18}$. For the ordinary wave (propagation perpendicular to $\vec{B}$ and $\vec{E} \| \vec{B})$ :

$$
\eta^{2}=1-\omega_{p}^{2} / \omega^{2}=1-n_{e} / n_{c}
$$

where $n_{c}=\omega^{2} m_{e} \varepsilon_{0} / e^{2}$ is the cutoff density. Thus

$$
\Delta \phi=\frac{\omega}{c} \int\left(\sqrt{1-n_{e} / n_{c}}-1\right) d l \simeq-\frac{\omega}{2 c n_{c}} \int n_{e} d l
$$

where the approximate equality holds when $n_{e} \ll n_{c}$. In standard interferometry analysis, it is customary to use the approximate expression; however, here we can apply the exact expression without additional difficulty since we use forward modelling. For the TJ-II microwave interferometer, $f=140 \mathrm{GHz}$, so $\omega=8.78 \cdot 10^{11} \mathrm{~s}^{-1}$ and $n_{c}=2.43 \cdot 10^{20} \mathrm{~m}^{-3}$.

For the purposes of this work, we rewrite the above expression as:

$$
\overline{n_{e}^{0}} \equiv-\frac{2 c n_{c} \Delta \phi}{\omega L_{c}}=\frac{1}{L_{c}} \int 2 n_{c}\left(1-\sqrt{1-n_{e}^{0}(R, \phi, Z) / n_{c}}\right) d l
$$

along the chord of the interferometer line of sight, parametrized as $\left(R_{\text {int }}(l), \phi_{\text {int }}, Z_{\text {int }}(l)\right)$, shown in Fig. 1. The chord length $L_{c}$ is the intersection of the chord with the plasma region $(\rho \leq 1)$ for the given configuration. Note that $\overline{n_{e}^{0}}$ is not simply the mean of $n_{e}^{0}$ along the chord, but includes the small correction for the dependence of the refractive index on $n_{e} / n_{c}$

discussed above. This computed value of $\overline{n_{e}^{0}}$ can be compared directly to the density signal $\left(\overline{n_{e}}\right)$ in the TJ-II database. While the density profile is allowed to extend beyond $\rho=1$, the normalization $L_{c}$ is still based on the intersection of the chord with the theoretical plasma region $(\rho \leq 1)$, since this is how the experimental signal is computed also.

The error of the phase detector is about $2 \pi / 10$, which translates into an error in the line-integrated density of about $\Delta{\overline{n_{e}}}^{\text {in }}=0.025 \cdot 10^{19} \mathrm{~m}^{-3}$ (assuming a typical chord length of $0.4 \mathrm{~m}$ and not considering spurious phase jumps), where the superscript "in" means "instantaneous". For the purposes of profile reconstruction, we will calculate the timeaveraged line integrated density by averaging the interferometry data over $1 \mathrm{~ms}$ of time (i.e., 


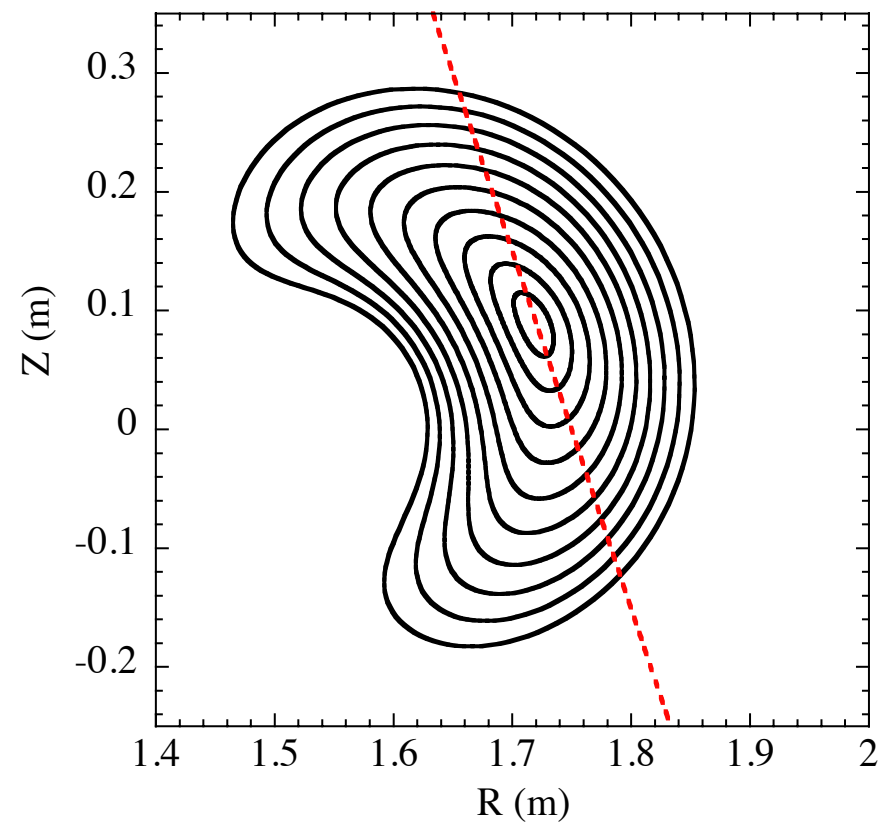

FIG. 1. Geometry of the $2 \mathrm{~mm}$ microwave interferometer beam in TJ-II Sector B8, $\phi=264.4^{\circ}$ (configuration shown: 100_44_64).

$N=100$ points). This time interval is long enough to smooth out rapid oscillations $\frac{19}{\text {, and }}$ short enough to adequately capture mean profile evolution. Therefore, the final error is $\Delta \overline{n_{e}}=\Delta \overline{n_{e}}$ in $/ \sqrt{N}=0.0025 \cdot 10^{19} \mathrm{~m}^{-3}$.

\section{Reflectometry}

For profile measurement, (X-mode) microwaves are launched as shown schematically in Fig. 2. For profile measurement, the beam is launched with a variable frequency $f$ (a ramp), and the return phase is detected ${ }^{20}$. The time delay $\tau^{0}(f)$ of the reflectometer beam, reflected off the cutoff layer inside the plasma, is estimated by:

$$
\tau^{0}(f)=\frac{1}{2 \pi} \frac{d \phi}{d f}
$$

where the phase is determined as:

$$
\phi(f)=\frac{4 \pi f}{c} \int_{r_{c}(f)}^{a} \eta(f, r) d r-\frac{\pi}{2}
$$

Here, $r_{c}(f)$ is the position of the cut-off layer (determined as the first point where $\eta$ becomes zero, counted from the plasma edge). The integration limit " $a$ " refers to the point where the 


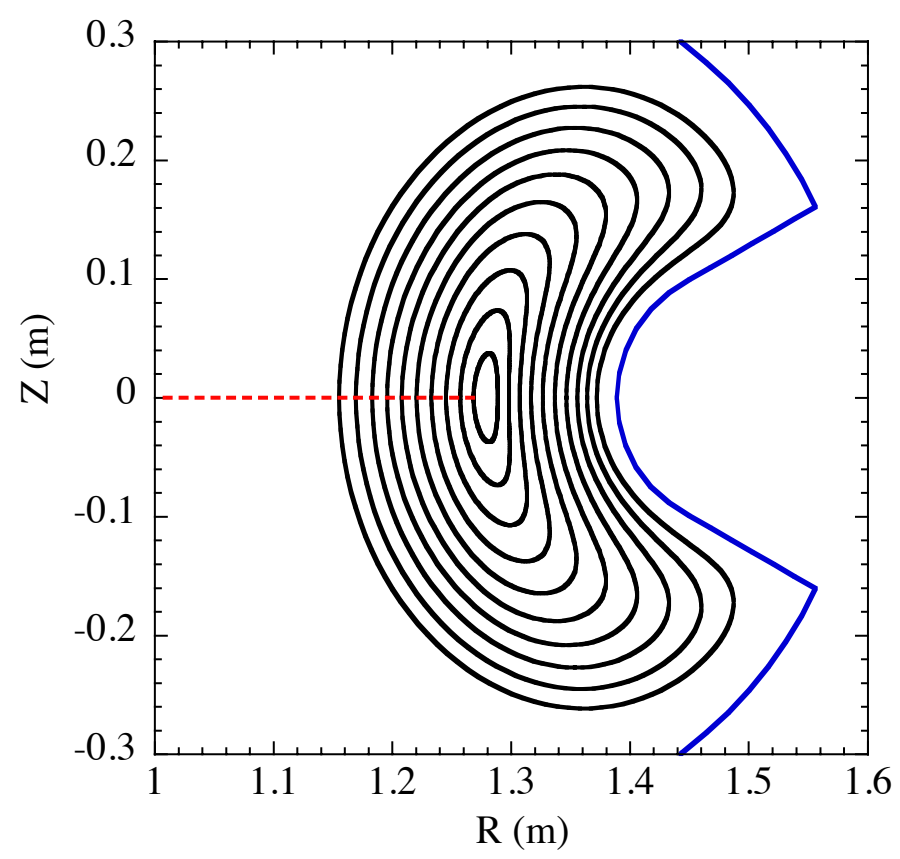

FIG. 2. Geometry of the reflectometer beam in TJ-II Sector A4, $\phi=135^{\circ}$ (configuration shown: 100_44_64). The location of the vacuum vessel is indicated by the blue line on the right.

density drops to zero; this point may be slightly outside the LCFS. The time delay can be used to reconstruct the density profile, as each frequency corresponds to a different cutoff position and density (cf. Appendix A).

In the above expression, the $\mathrm{X}$-mode refractive index $\eta(f, r)$ is computed using $(\omega=2 \pi f)$ :

$$
\begin{aligned}
\eta^{2} & =1-\frac{X(1-X)}{1-X-Y^{2}} \\
X & =\omega_{p e}^{2} / \omega^{2} \\
Y & =\omega_{e c} / \omega \\
\omega_{p e}^{2} & =n_{e}^{0} e^{2} / \varepsilon_{0} m_{e} \\
\omega_{e c} & =e B / m_{e}
\end{aligned}
$$

Thus, the expected time delay $\tau^{0}(f)$ can be computed from the model profile $n_{e}^{0}(\rho)$. The above expression only contemplates propagation inside the plasma (the region where $n_{e}^{0}(\rho)>$ $0)$. The vacuum correction is discussed below.

The measurements do not deliver the time delay $\tau(f)$ directly. In actual fact, the reference beam is amplitude modulated with a modulation frequency $f_{\text {mod }}$. The spectrum of the beam 
is therefore characterized by $f$ and two sidebands at $f \pm f_{\text {mod }}$, while $f$ is scanned. The detection equipment delivers two quadrature (sine and cosine) signals $s(t)$ and $c(t)$, as well as the reference frequency $f(t)$. The calculation to deduce $\tau(f)$ from these raw data involves some careful smoothing and correction of phase jumps.

The instantaneous signal amplitude $A(t)$ is calculated as $A(t)=\sqrt{c^{2}+s^{2}}$ and the instantaneous phase as $\phi(t)=\tan ^{-1}(s / c)$. Assuming a random and independent measurement error of size $\epsilon$ in the measured signals $c$ and $s$, the error in $A$ is $\Delta A=\epsilon$, while the error in $\phi$ is $\Delta \phi=\epsilon / A$ (using standard error propagation). This approximate result must be corrected for the non-Gaussianity of the error distribution of $c$ and $s$ due to the non-linearity of the trigonometric functions. It can be shown that the above calculations underestimate the error in $\phi$ by a factor of $\sqrt{2}$ (see Appendix B). To correct for this, we will estimate $\Delta \phi$ by $\Delta \phi=\sqrt{2} \epsilon / A$.

From the above considerations, $\epsilon$ can be estimated from the Root Mean Square (RMS) deviation of $A(t)$ for actual data. For this purpose $\operatorname{RMS}(A)$ is calculated using 31-point bins. The experimental time delay is obtained from $\tau=\phi / 2 \pi f_{\bmod } 2122$, where $f_{\bmod }$ is the modulation frequency $(0.2 \mathrm{GHz})$ of the carrier frequency. The corresponding error in $\tau$ is thus found from $\Delta \tau=\Delta \phi / 2 \pi f_{\text {mod }} \simeq \sqrt{2} \epsilon / 2 \pi f_{\bmod } A=\operatorname{RMS}(A) \sqrt{2} / 2 \pi f_{\text {mod }} A$. This estimate adequately takes account of the effects of occasional signal loss (leading to small amplitudes A) during a frequency scan 21 .

The final measured time delay $\tau_{\text {ref }}$ is calibrated by subtracting the vacuum time delay (the delay corresponding to the microwaves bouncing off the vacuum vessel wall, located at $R=R_{\text {wall }}$, see Fig. 22 from the measured time delay: $\tau_{\text {ref }}(f)=\tau(f)-\tau_{\text {vac }}(f)$. This calibration is important, as it removes any spurious time delays caused by propagation in waveguides and other parts of the measurement equipment. The vacuum correction is assumed not to contribute significantly to the measurement error, so $\Delta \tau_{\text {ref }}=\Delta \tau$. To calibrate the modelled $\tau^{0}(f)$ in the same way, an offset $\tau_{\text {off }}$ is added that is calculated from the profile $n_{e}^{0}(\rho)$ as

$$
\tau_{\text {off }}=2\left(R\left(n_{e}^{0}=0\right)-R_{\text {wall }}\right) / c
$$

yielding a typical value of about $-1.5 \mathrm{~ns}$ (modulo $5 \mathrm{~ns}$ in view of the modulation frequency $\left.f_{\text {mod }}=0.2 \mathrm{GHz}\right)$. 


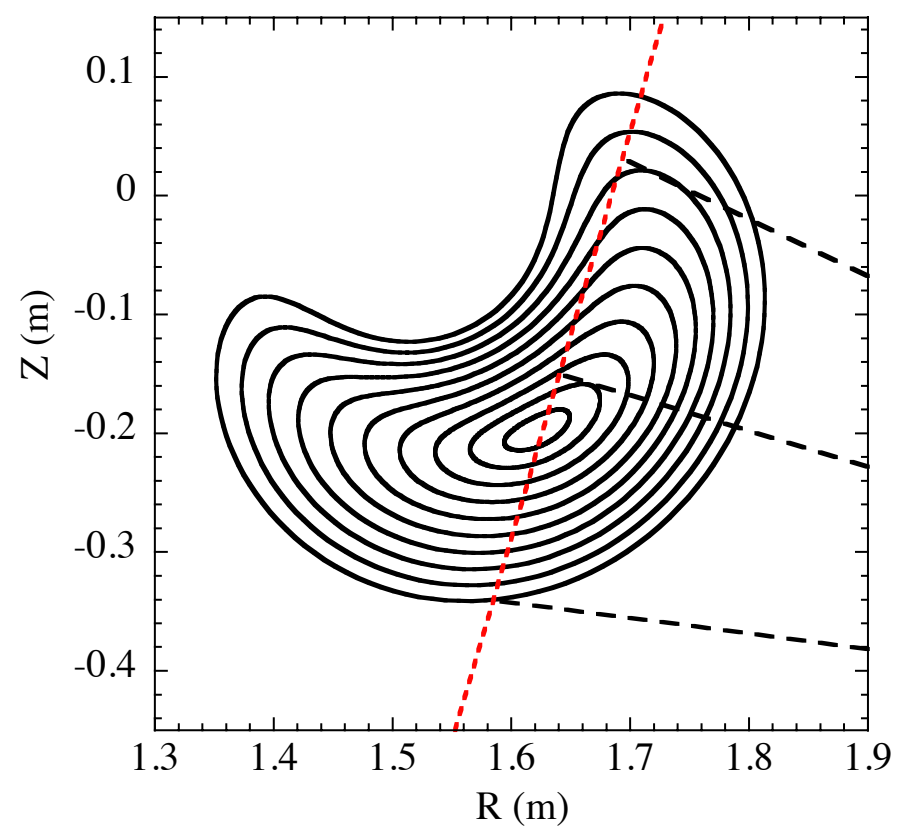

FIG. 3. Geometry of the Thomson Scattering probing laser beam (red dashed line) in TJ-II Sector D2, $\phi=14.5^{\circ}$ (configuration shown: 100_44_64). The observation optics (the spectrometer) are located to the right (some lines of sight are indicated by long-dashed lines).

\section{E. Thomson Scattering}

The Thomson Scattering diagnostic provides local measurements of $n_{e}$ and $T_{e}$, with a high radial resolution of between 2 and $6 \mathrm{~mm}^{23}$. Fig. 3 shows the geometry of the probing laser beam and some of the viewing chords. The present work relies on the reconstruction of the local profile $n_{e}\left(\rho_{i}\right)$ from the measured spectrum, along with the corresponding errors, $\Delta n_{e}\left(\rho_{i}\right)\left(i=1, \ldots, N_{T h}\right)$, delivered by standard data analysis software described elsewhere ${ }^{23}$. This information is retrieved from the TJ-II shot database ${ }^{24}$. Due to drifts in the absolute calibration of the diagnostic, an additional scale parameter $A_{T h} \simeq 1$ will be used to multiply the TS density profile (see below). The Thomson Scattering laser can be fired only once during the discharge. Therefore, profile reconstructions using the TS data are limited, in principle, to times close to the TS laser pulse time (and, due to a fixed delay between the software trigger and the actual firing of the laser, the actual Thomson Scattering laser pulse time is the time stored in the database $+6.4 \mathrm{~ms})$. However, the scaling factor $A_{T h}$ allows some flexibility in this sense: provided plasma conditions and the shape of the density profile 


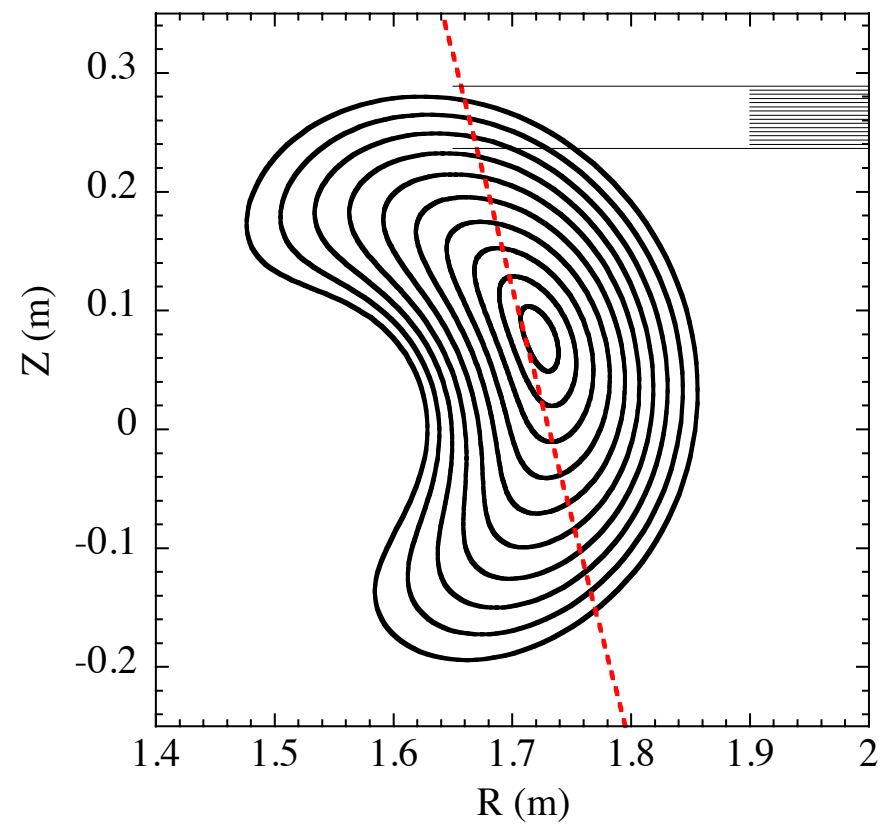

FIG. 4. Geometry of the supersonic Helium beam trajectory in TJ-II Sector C8, $\phi=355.3^{\circ}$ (dashed line, injection from above; configuration shown: 100_44_64). The observation optics are located to the right; the 16 observation lines are indicated schematically, and the first and last line are prolonged up to their intersection with the Helium beam to indicate the observation region.

are known not to change much between the laser time $t_{T h}$ and some other time of interest $t_{1}$, the scale factor of the profile is adjusted automatically (using the line-integrated density from the interferometer as a reference) to provide an approximate profile shape at $t_{1}$, some time away from the laser pulse time, as discussed below.

\section{F. Helium beam}

The Helium beam diagnostic consists of a supersonic Helium source with a pulsed valve system and a system for the detection of the resulting line emission 2526 . Fig. 4 shows the geometry of the probing supersonic Helium beam. The Helium beam can be operated in pulsed mode, with a repetition rate of up to $200 \mathrm{~Hz}$, thus providing profiles every $5 \mathrm{~ms}$ or more. The observation system detects three emission lines $\left(\lambda_{j}=667,706\right.$, and $728 \mathrm{~nm}$ for $j=1, \ldots, 3)$ along $N_{H e}=16$ lines of sight, nearly perpendicular to the Helium beam. From the ratio of the line amplitudes, $n_{e}$ and $T_{e}$ can be deduced using an interpolated table lookup 


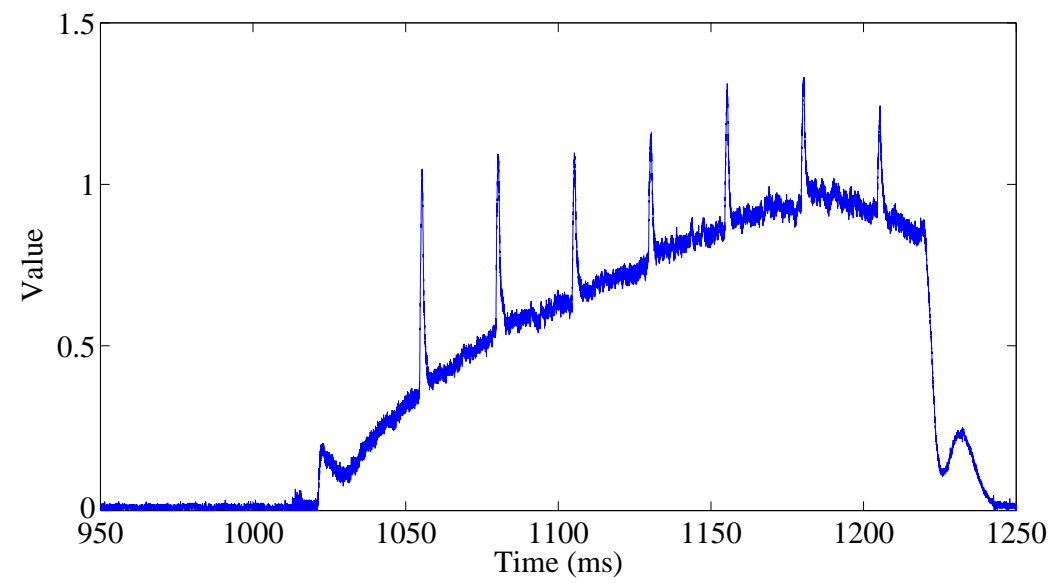

FIG. 5. Shot 24729, example time trace of the He line emission, channel 06, emission line at 667 nm.

procedure, using the tabulated values of predicted line ratios versus density and temperature, based on a collisional-radiative model 26 . This model has been shown to provide a satisfactory accuracy for typical TJ-II plasmas, but is subject to future improvement. Due to the crossing of the Helium beam and the lines of sight, this diagnostic provides local measurements (with a radial resolution of about $4 \mathrm{~mm}$ and a toroidal resolution of about $20 \mathrm{~mm}$ ), limited to the edge region due to beam attenuation.

An example of the evolution of the line emissions in shown in Fig. 5. There is a gradual buildup of emission, and on top of that the pulses are visible. The variance of the signal is seen to increase proportionally to the total emission intensity, which is typical of Poisson noise (or "shot noise"). The control signal from the beam valve allows identifying the "beam on' time interval closest to the time of interest. The subsequent analysis is limited to a time interval, centered around this 'beam on' time, containing both the pulse itself and the background. One of these pulses is selected for analysis and a time window is chosen, centered on the selected pulse, such that it includes only this pulse. In the following, we describe the analysis procedure applied to each of the $3 \times 16=48$ detected line emission signals in the selected time window.

The actual measurement $I_{\text {act }}(t)$, in appropriate units, is related to the measured emission $I_{\text {meas }}$ via a scale factor $S>0$ :

$$
I_{\text {act }}(t)=S I_{\text {meas }}(t)
$$

This scale factor is needed because the measured emission is in arbitrary units, not in 
photon count units. $I_{\text {meas }}$ is offset-corrected by determining the mean value at the start of the discharge (prior to He injection) and subtracting this value from the data.

We assume that the detected line waveform can be modelled, to good approximation, by:

$$
I(t)=S\left(I_{b}(t)+A G\left(t-t_{0}, \sigma\right)\right),
$$

where $G$ is a normalized Gaussian, $G(t, \sigma)=\exp \left(-t^{2} / 2 \sigma^{2}\right) / \sqrt{2 \pi \sigma^{2}}$. The line emission intensity $I(t)$ is a positive definite quantity because it is due to a photon emission process, and subject to Poisson statistics. The term $I_{b}$ defines the background emission, the level of which is important in the framework of Poisson statistics. In a neighbourhood of a given emission peak, this background is modelled by a linear drift:

$$
I_{b}(t)=c+b t
$$

Below we will show how the background drift parameters, the peak time $t_{0}$, and the scale factor $S$ are determined.

Assuming Poisson statistics, the probability of measuring an actual emission $I_{\text {act }}(t)$, given the model line intensity $I(t)$, is:

$$
p\left(I_{\text {act }}(t) \mid I(t)\right)=\frac{I(t)^{I_{\text {act }}(t)} e^{-I(t)}}{\Gamma\left(I_{\text {act }}(t)+1\right)}
$$

This allows us to define a logarithmic likelihood function $L$ :

$$
L(\vec{\alpha})=\frac{1}{N} \sum_{i=1}^{N} \log p\left(I_{\text {act }}\left(t_{i}\right) \mid I\left(t_{i}\right)\right)
$$

where the parameter vector $\vec{\alpha}$ consists of: $\{A, \sigma\}$; the remaining parameters $\left\{t_{0}, b, c, S\right\}$ are not varied and are determined as described below. The best fit is obtained by maximizing $L$, and the error in the parameters can be found from the shape of $L$ near its maximum.

The background drift is determined by fitting the first and last $20 \%$ of the data, denoted by $I_{\text {meas }}^{\text {back }}$ (consisting of two disconnected time intervals, centered at the peak) to a straight line, yielding $b$ and $c$ directly (different values for each emission line).

To find $t_{0}$, the data from the 48 data channels ( 3 line emissions at 16 positions) are summed together (yielding $I_{\text {meas }}^{\text {tot }}\left(t_{i}\right)$ ), and $t_{0}$ is found from

$$
t_{0}=\sum_{i} t_{i}\left|I_{\text {meas }}^{\mathrm{tot}}\left(t_{i}\right)\right| / \sum_{i}\left|I_{\text {meas }}^{\mathrm{tot}}\left(t_{i}\right)\right|
$$




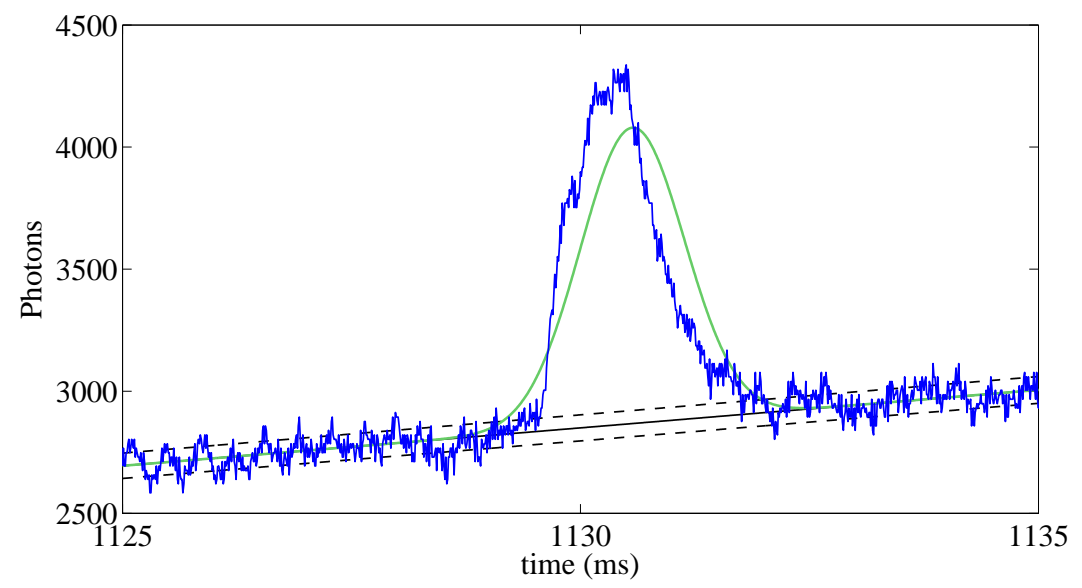

FIG. 6. Shot 24729, scaled He line emission, channel 06, emission line at $667 \mathrm{~nm} I_{\text {act }}$ (blue), and fit $I$ (green Gaussian). The width of the Poisson distribution (the error level) is indicated by the three slanted parallel lines.

Allowing $t_{0}$ to be a free parameter would lead to very bad fits for channels with a low signal to noise ratio.

The scale factor $S$ is determined from the requirement that the emission should follow Poisson statistics. This means that the variance of the background should be related to its mean by the following relation:

$$
\left\langle I_{\mathrm{act}}^{\mathrm{back}}\right\rangle=\operatorname{var}\left(I_{\mathrm{act}}^{\mathrm{back}}\right)
$$

where the angular brackets indicate the mean value, and "var" stands for "variance". Thus, using Eq. (17):

$$
S=\left\langle I_{\text {meas }}^{\text {back }}\right\rangle / \operatorname{var}\left(I_{\text {meas }}^{\text {back }}\right)
$$

In Fig. 6 we show an example of a fit to the line emission. Shown are: $I_{\text {act }}$, $I$, the background drift, and the (Poisson) error level of the background. The vertical axis indicates the photon counts. The misfit of the line shape is due to the fact that the model waveform (a symmetric Gaussian) is not completely adequate, as the line emission waveform rises quicker than it decays; its actual waveform consists of an exponential rise $\left(\propto 1-\exp \left(-\left(t-t_{1}\right) / \tau\right)\right)$ followed by an exponential decay $\left(\propto \exp \left(-\left(t-t_{2}\right) / \tau\right)\right)$. However, since we are only interested in the relative amplitudes of the waveform, the Gaussian is a satisfactory approximation.

The errors in $A_{i j}, i=1, \ldots, 16$ indicating the positions and $j=1, \ldots, 3$ the three emission lines, are propagated to the ratios $R_{i j}$ using standard error propagation. The line ratios are 
computed as $R_{i 1}=C_{1} A_{i 1} / A_{i 3}$ and $R_{i 2}=C_{2} A_{i 3} / A_{i 2}$, where the $C_{k}(k=1,2)$ are calibration factors, each with corresponding error $\Delta C_{k}$. Therefore:

$$
\left(\frac{\Delta R_{i 1}}{R_{i 1}}\right)^{2}=\left(\frac{\Delta C_{1}}{C_{1}}\right)^{2}+\left(\frac{\Delta A_{i 1}}{A_{i 1}}\right)^{2}+\left(\frac{\Delta A_{i 3}}{A_{i 3}}\right)^{2}
$$

and similar for $R_{i 2}$.

The error $\Delta n_{e}$ can easily be deduced applying straightforward error propagation to the interpolated table lookup procedure ${ }^{26}$. The table lookup yields $n_{e}\left(\rho_{i}\right)=f\left(R_{i 1}, R_{i 2}\right)$ and $T_{e}\left(\rho_{i}\right)=g\left(R_{i 1}, R_{i 2}\right)$, so the propagated error is

$$
\left(\Delta n_{e}\left(\rho_{i}\right)\right)^{2}=\left(\frac{\partial f\left(R_{i 1}, R_{i 2}\right)}{\partial R_{i 1}} \Delta R_{i 1}\right)^{2}+\left(\frac{\partial f\left(R_{i 1}, R_{i 2}\right)}{\partial R_{i 2}} \Delta R_{i 2}\right)^{2}
$$

and similar for $T_{e}\left(\rho_{i}\right)$. Here, the partial derivatives are computed using finite differences with step size equal to the errors in $R_{i j}$, since the error is not infinitesimal.

\section{RECONSTRUCTION OF THE TJ-II DENSITY PROFILE}

As explained in Section II, the posterior probability distribution is a product of probabilities, $\mathcal{L}=\Pi_{j} \mathcal{L}_{j}$, each factor corresponding to either a prior or a diagnostic (forward model). We define the logarithmic posterior $L=\log \mathcal{L}=\sum_{j} L_{j}$. In the following, we define the form of the individual terms of this sum used in the reconstruction of the density profile.

\section{A. Priors}

In order to guarantee physically reasonable profiles, a number of priors is used.

To guarantee positivity of the profile in the region $0 \leq \rho \leq 1$, we introduce a term

$$
L_{1}=\sum_{i=1}^{n_{1}}-\frac{\left(\min \left(0, n_{e}^{0}\left(\rho_{i}\right)\right)^{2}\right.}{2 \sigma_{1}^{2}}
$$

The $\rho_{i}$ are chosen equidistant in the range $0 \leq \rho_{i} \leq 1$. We choose $n_{1}=20$, and $\sigma_{1}=0.0001$, yielding a high penalty for negative profiles. A positive (acceptable) profile has $L_{1}=0$ (i.e., probability $\left.P_{1}=\exp \left(L_{1}\right)=1\right)$.

Another term is introduced to keep the density $n_{e}^{0}(\rho)$ small outside the LCFS $(\rho>1)$ :

$$
L_{2}=\sum_{i=1}^{n_{2}}-\frac{\left(\max \left(0, n_{e}^{0}\left(\rho_{i}\right)\right)^{2}\right.}{2 \sigma_{2}^{2}}
$$


Negative densities are ignored. The $\rho_{i}$ are chosen equidistant in the range $1.1 \leq \rho_{i} \leq 1.2$, while $n_{2}=4$ and $\sigma_{2}=0.01$. This prior allows a non-zero density close to the LCFS $(\rho<1.1)$, but penalizes positive densities further away.

A third prior is introduced to reflect the knowledge, obtained from experience, that density profiles tend to be rather flat in the centre:

$$
L_{3}=\sum_{i=1}^{n_{3}}-\frac{\left(\partial_{\rho} n_{e}^{0}\left(\rho_{i}\right)\right)^{2}}{2 \sigma_{3}^{2}}
$$

where $\partial_{\rho} f=\partial f / \partial \rho$. The $\rho_{i}$ are chosen equidistant in the range $0 \leq \rho_{i} \leq 0.6$, while $n_{3}=5$ (typically) and $\sigma_{3}=0.1$. This prior is only used when a reconstruction is made without information from Thomson Scattering (so that no profile information is available for the central part of the profile).

The choice of values for $n_{1}, n_{2}$, and $n_{3}$ is somewhat arbitrary and motivated by a practical balance between a sufficient sampling of the solution space and reduced computational effort. It is possible that specific circumstances may require more priors. For example, oscillating profiles can be avoided by penalizing second derivatives. In the present work we will not consider this possibility.

\section{B. Interferometry}

As explained above, the interferometer provides $\overline{n_{e}}$ and a corresponding error $\Delta \overline{n_{e}}$. Thus, we add the corresponding term to $L$ :

$$
L_{4}=-\frac{\left(\overline{n_{e}}-\overline{n_{e}^{0}}\right)^{2}}{2\left(\Delta \overline{n_{e}}\right)^{2}}
$$

\section{Reflectometry}

Another term corresponds to reflectometry:

$$
L_{5}=-\frac{N_{\text {fr }}}{N_{\text {ref }}} \sum_{i=1}^{N_{\text {ref }}} \frac{\left(\tau_{\text {ref }}(i)-\tau^{0}(i)-\tau_{\text {off }}\right)^{2}}{2\left(\Delta \tau_{\text {ref }}(i)\right)^{2}}
$$

Here, the index $i$ enumerates the points of the reflectometer frequency scan around the time of analysis (typically, $N_{\text {ref }} \simeq 1000$, covering $2 \mathrm{~ms}$ of real time). Time evolution of the profiles during the scan is not considered. The calibration parameter $\tau_{\text {off }}$, calculated according to 
Eq. (16), is explicitly included in the expression. The error $\Delta \tau_{\text {ref }}$ is estimated using the method described above.

The factor $N_{\text {fr }} / N_{\text {ref }}$ arises due to the fact that the time delay $\tau\left(f_{r}\right)$ is computed from an amplitude-modulated signal with modulation frequency $f_{\text {mod }}=0.2 \mathrm{GHz}$. The reference frequency $f_{r}$ is scanned from $f_{r}^{0} \simeq 25 \mathrm{GHz}$ to $f_{r}^{1} \simeq 50 \mathrm{GHz}$ while its amplitude is being modulated, and during the scan $N_{\text {ref }}$ measurements $\tau\left(f_{r}\right)$ are obtained. However, samples that are less than (twice) the modulation frequency $f_{\text {mod }}$ apart are not statistically indepen-

dent: thus, in the range $f_{r}^{0} \leq f_{r} \leq f_{r}^{1}$, only $N_{\text {fr }}=\left(f_{r}^{1}-f_{r}^{0}\right) / 2 f_{\text {mod }}$ independent samples are obtained (the factor 2 appears because the spectrum of the AM signals contains two peaks at $f_{r} \pm f_{\text {mod }}$, separated by $\left.2 f_{\text {mod }}\right)$. Typically, this means that the $\sim 1000$ actual measurements only contain $\sim 60$ independent information units.

\section{Thomson Scattering}

As mentioned above, Thomson Scattering is handled by incorporating the reconstructed TS profile $n_{e}^{T h}(\rho)$ along with its error $\Delta n_{e}^{T h}(\rho)$. Thus, adding Thomson Scattering comes down to adding the following term to $L$ :

$$
L_{6}=-\sum_{i=1}^{N_{T h}} \frac{\left(A_{T h} n_{e}^{T h}\left(\rho_{i}\right)-n_{e}^{0}\left(\rho_{i}\right)\right)^{2}}{2\left(A_{T h} \Delta n_{e}^{T h}\left(\rho_{i}\right)\right)^{2}}
$$

where a TS scaling factor $A_{T h}$ has been included as an additional free parameter without restrictions (in other words, the prior distribution for $A_{T h}$ is flat).

\section{E. Helium beam}

Similar to the above, adding Helium beam information amounts to adding the following term to $L$ :

$$
L_{7}=-\sum_{i=1}^{N_{H e}} \frac{\left(n_{e}^{H e}\left(\rho_{i}\right)-n_{e}^{0}\left(\rho_{i}\right)\right)^{2}}{2\left(\Delta n_{e}^{H e}\left(\rho_{i}\right)\right)^{2}}
$$




\section{F. Total posterior probability}

The total posterior probability is then

$$
L=\sum_{i=1}^{7} \theta_{i} L_{i}
$$

where $\theta_{i}$ are switches $\left(\theta_{i}=0,1\right)$ that allow selecting priors and diagnostics. The best profile $n_{e}^{0}(\rho)$ is found by varying the $\alpha_{k}$ (and $A_{T h}$, in case TS is used) in order to maximize $L$. This is done using a standard routine $\left(\mathrm{E} 04 \mathrm{FYF}^{27}\right)$. A major problem of this approach is that the maximization may get stuck in a local maximum, so that the result may depend on the initial set of parameters at the start of the maximization (which is undesirable). The starting point of the maximization is usually taken from a previous run, as profiles tend to have roughly the same global shape. Otherwise, a safe procedure is starting from a very low value of $K$ (the number of profile parameters) and gradually increasing $K$ to its final value over successive runs.

The inclusion of more priors may alleviate the sensitivity to initial conditions and local maxima, as it delimits the solution space. In any case, the random exploration of parameter space involved in marginalization process (see below) allows detecting other local maxima of $L$, if any, and reinitializing the maximum search from such a new, higher maximum.

\section{G. Error estimation by marginalization}

Once we know the 'best' profile $n_{e}^{0}(\rho)$ from the maximization of $L$, we would also like to know its error $\Delta n_{e}^{0}(\rho)$. This is done on the basis of the joint probability distribution

$$
P\left(n_{e}^{0}, \vec{\alpha} \mid \vec{d}, \vec{\sigma}, I\right)
$$

where $n_{e}^{0}$ is the model profile, $\vec{d}$ are the data, and $\vec{\sigma}$ the errors in the data. The model parameters are represented by $\vec{\alpha}$.

Then, the mean and mean square values of the density are determined by

$$
\begin{array}{r}
\overline{n_{e}^{0}}=\int n_{e}^{0} P\left(n_{e}^{0}, \vec{\alpha} \mid \vec{d}, \vec{\sigma}, I\right) d \vec{\alpha} \\
\overline{\left(n_{e}^{0}\right)^{2}}=\int\left(n_{e}^{0}\right)^{2} P\left(n_{e}^{0}, \vec{\alpha} \mid \vec{d}, \vec{\sigma}, I\right) d \vec{\alpha}
\end{array}
$$


which can be simplified by means of the product rule

$$
P\left(n_{e}^{0}, \vec{\alpha} \mid \vec{d}, \vec{\sigma}, I\right)=P\left(n_{e}^{0} \mid \vec{\alpha}, \vec{d}, \vec{\sigma}, I\right) \cdot P(\vec{\alpha} \mid \vec{d}, \vec{\sigma}, I)
$$

where the first term on the right-hand side is a delta function, since $\vec{\alpha}$ completely specifies $n_{e}^{0}$. The second term is proportional to the posterior probability specified above. The proportionality factor is important, but can be obviated by normalizing the expressions Eq. (36) by

$$
\int P\left(n_{e}^{0}, \vec{\alpha} \mid \vec{d}, \vec{\sigma}, I\right) d \vec{\alpha}
$$

From this, the error in the model or reconstructed density $\Delta n_{e}^{0}$ is found from

$$
\left(\Delta n_{e}^{0}\right)^{2}=\overline{\left(n_{e}^{0}\right)^{2}}-\left(\overline{n_{e}^{0}}\right)^{2}
$$

The multi-dimensional integral over the parameters $\alpha_{i}$ is performed using Monte Carlo estimation with importance weighting. For the purpose of distributing the random sampling points needed for this procedure, the shape of the function $L(\vec{\alpha})$ near its maximum, $L\left(\vec{\alpha}^{0}\right)$, is approximated by a parabolic function with negative curvature in all directions (i.e., this distribution is approximated by a product of Gaussians, one for each coordinate direction $\left.\alpha_{i}\right)$. In order to estimate the width $\sigma\left(\alpha_{i}\right)$ of the Gaussian in a given direction $\alpha_{i}, L$ is sampled around its maximum. Since no information about the numerical size of $\sigma\left(\alpha_{i}\right)$ is available a priori, $\alpha_{i}$ is varied logarithmically to explore all possible relevant scales, taking 21 points distributed as $\alpha_{i}(s)=\alpha_{i}^{0}+\operatorname{sgn}(s) \cdot 10^{|s|-6}, s=-10,-9, \ldots, 10$, where $\operatorname{sgn}(s)$ is the $\operatorname{sign}$ function $(\operatorname{sgn}(s)=1$ for $s>0, \operatorname{sgn}(s)=-1$ for $s<0$, and $\operatorname{sgn}(0)=0)$. The zeroth $\left(M_{i, 0}\right)$ and second $\left(M_{i, 2}\right)$ statistical moments of $\exp (L)$ are computed as:

$$
M_{i, j}=\int_{\alpha_{i}}\left(\alpha_{i}-\alpha_{i}^{0}\right)^{j} \exp (L(\vec{\alpha}, \ldots)) d \alpha_{i}
$$

keeping all $\alpha_{j}, j \neq i$ fixed at their optimal value $\alpha_{j}^{0}$. Account is taken of the non-homogeneous distribution of the sampling points $\alpha_{i}(s)$ when evaluating this integral as a sum over the 21 sampled values. $\sigma\left(\alpha_{i}\right)$ is estimated from $\sqrt{M_{i, 2} / M_{i, 0}}$.

Note that $\sigma\left(\alpha_{i}\right)$ need not be very precise, since the actual marginalization is done using the true function $L$. So, when using the Monte Carlo approach to evaluate the multi-dimensional integral, the random values of $\vec{\alpha}$ are distributed according to a product of Gaussians, centered at the maximum likelihood point $\vec{\alpha}^{0}$, and with a width along each parameter axis given by 
$\sigma\left(\alpha_{i}\right)$. The moments $(36)$ and $(38)$ are then found as sums over the random instances of $\vec{\alpha}$, corrected for the Gaussian weights 28 . We use 1000 random points to evaluate the integrals.

If a higher maximum is encountered during the evaluation of the marginalization integrals, then the process of maximization and subsequent marginalization can be restarted from that new point. This technique significantly reduces the probability of 'getting stuck' in a local maximum.

\section{H. Error interpretation and local profile error}

While the marginalization process of the preceding section provides an error estimate of the final profile, some remarks regarding its interpretation are necessary.

Assume we perform a hypothetical profile measurement that returns profile estimates $n_{e}\left(\rho_{i}\right)$ for a set of points $i=1, \ldots, N$. As described above, we model this profile by a function $n_{e}^{0}(\rho, \vec{\alpha})$ with $K$ degrees of freedom, i.e., the parameter vector $\vec{\alpha}$ has length $K$.

The corresponding (global) logarithmic likelihood is given by:

$$
L(\vec{\alpha})=\sum_{i=1}^{N} \log p\left(n_{e}\left(\rho_{i}\right) \mid n_{e}^{0}\left(\rho_{i}, \vec{\alpha}\right)\right),
$$

where $p$ is the appropriate likelihood distribution for the individual measurements. For simplicity, we assume the $\rho_{i}$ are given by, e.g., $\rho_{i}=i / N$ (uniformly distributed). As $N$ is increased (by performing more measurements), the function $L(\vec{\alpha})$ becomes ever narrower, corresponding to the continuous reduction of the error in $\vec{\alpha}$. This makes sense, because adding measurements means that the knowledge of $\vec{\alpha}$ improves and the error in $\vec{\alpha}$ is reduced.

However, if we now attempt to determine the error in the density profile by marginalization over $\vec{\alpha}$, the error in the (local) density also decreases with $N\left(\Delta n_{e}^{0} \propto 1 / \sqrt{N}\right)$. This correctly reflects the improved knowledge of the parameters $\vec{\alpha}$, but does not reflect our expectations regarding the "local precision" of the profile $n_{e}^{0}(\rho)$. As an example, additional measurements near $\rho=1$ help to define the global profile (improving the knowledge of $\vec{\alpha}$ ), but should not affect the precision of the profile at a remote location, e.g., near $\rho=0$. This would only make sense if the profile was totally "stiff", e.g., a fixed radial shape multiplied by a variable constant $(K=1)$. But if $K>1$, the errors in $n_{e}$ near $\rho=0$ and $\rho=1$ should be essentially decoupled. In fact, one would expect the final error of the density $n_{e}^{0}\left(\rho_{i}\right)$ to be 
close to $\Delta n_{e}\left(\rho_{i}\right)$ (the local measurement error of $n_{e}\left(\rho_{i}\right)$ ), and in particular, the local error should be essentially independent from $N$.

To estimate this local error in $n_{e}^{0}\left(\rho_{i}\right)$, we can introduce a local version of the global logarithmic likelihood, or (formally):

$$
L_{\mathrm{loc}}(\vec{\alpha}, \rho)=\sum_{i=1}^{N} \log p\left(n_{e}\left(\rho_{i}\right) \mid n_{e}^{0}\left(\rho_{i}, \vec{\alpha}\right)\right) \delta\left(\rho_{i}-\rho\right)
$$

such that $L(\vec{\alpha})=\int L_{\mathrm{loc}}(\vec{\alpha}, \rho) d \rho$. The local likelihood only responds to profile variations at a given value of $\rho$ and thus measures the local profile error. Obviously, the formal expression (with a delta function) is not practical due to the limited spatial resolution of the measurements; we will therefore use a test function with a finite width $\sigma_{\text {test }}$, as follows.

The sensitivity of the likelihood to local profile modifications can be explored by modifying $n_{e}^{0}(\rho)$ in a small neighbourhood of a given radius $\rho_{\text {test }}$, e.g., by adding a test function to the profile equal to a narrow Gaussian, centered at $\rho_{\text {test }}$, and observing the variation of $L$. The amplitude of this variation can then be used to define the local error bar. Thus, we replace $n_{e}^{0}(\rho)$ by:

$$
n_{e}^{0}(\rho) \rightarrow n_{e}^{0}(\rho)+\Delta n_{\text {test }} \exp \left(-\frac{\left(\rho-\rho_{\text {test }}\right)^{2}}{2 \sigma_{\text {test }}^{2}}\right)
$$

We then choose various values of $\Delta n_{\text {test }}$, and record the likelihood response $L\left(\Delta n_{\text {test }}\right)$, keeping $\vec{\alpha}$ fixed at the optimum value. The width of this response distribution (i.e., the value of $\Delta n_{\text {test }}$ for which $L$ changes by 1) defines the local profile error at $\rho=\rho_{\text {test }}$. A similar procedure has also been discussed by Fischer ${ }^{17}$. Obviously, the local profile error depends on the test function width $\sigma_{\text {test }}$; in the following, we will use $\sigma_{\text {test }}=0.01$ as a compromise between a test function that is as narrow as possible, yet sufficiently wide to generally include several measurement points. So the reported error bar can be understood as the local profile precision, where "local" is defined by an interval with full width $2 \sigma_{\text {test }}$.

A similar procedure is used to explore the local precision of $d n_{e}^{0}(\rho) / d \rho$; namely by modifying the profile by

$$
n_{e}^{0}(\rho) \rightarrow n_{e}^{0}(\rho)+\Delta n_{\text {test }}^{\prime}\left(\rho-\rho_{\text {test }}\right) \exp \left(-\frac{\left(\rho-\rho_{\text {test }}\right)^{2}}{2 \sigma_{\text {test }}^{2}}\right)
$$




\section{PROFILE RECONSTRUCTION EXAMPLES}

In the following, we present a few examples of the application of the method to density profile reconstruction at TJ-II.

In Section VA, we show how the Bayesian method is capable of robustly reconstructing the density profile using various combinations of diagnostics. It is shown how the precision of the result depends on the available diagnostics, and how a missing diagnostic (Thomson scattering) can be handled by activating an appropriate prior. This is important in view of the fact that the TJ-II Thomson scattering system is a single-pulse system.

In Section VB, we present a case with electrode biasing (leading to enhanced particle confinement), showing a robust and automated reconstruction of the density profile is possible based on reflectometry and interferometry, and allowing the visualization of the dynamical evolution of the density profile across a confinement transition - something that was hard or impossible to do using the traditional (non-robust) reflectometry profile reconstruction method.

Finally, in Section VC, we present density profile reconstructions across an L-H transition in order to show that the method is also suitable for high density discharges.

\section{A. An ECRH plasma}

The profile reconstruction program is modular and allows selecting which diagnostics are used (by means of the parameters $\theta_{i}$ ), so that the effect of adding diagnostics on profile reconstruction can easily be observed. In this section, we analyze a typical ECRH plasma (discharge 24729, magnetic configuration 100_44_64, $\overline{n_{e}} \simeq 0.6 \cdot 10^{19} \mathrm{~m}^{-3}$ ). Table II gives details about 4 cases studied (switching on and off diagnostics), and Figs. 7 , 10 show the corresponding profiles. The absolute value of $L$ listed in the table may seem rather large. However, to put these numbers into perspective, it should be noted that the effective number of terms contributing to $L$ is about 60 (reflectometry), 200 (Thomson Scattering), and 16 (Helium beam), plus 25-30 additional terms (priors and interferometry). A corresponding value of the reduced $\chi^{2}$ (a standard tool to judge the quality of least-squares fits) can be defined by $\chi^{2}=-2 L / N_{\text {tot }}$, where $N_{\text {tot }}$ is the total number of terms. Thus, the obtained values of $L$ correspond to reduced $\chi^{2}$ values of the order of 1 , i.e., reasonably good fits. This 
situation also serves to confirm that the errors are defined properly.

In the following figures, the reconstructed profile corresponding to the maximum likelihood point is indicated by a black line, and the local one- $\sigma$ error level (computed as described in the preceding section with $\left.\sigma_{\text {test }}=0.01\right)$ is indicated by a grey area.

Fig. 7 shows the reconstruction based on the reflectometry and interferometry data alone. Since no data are available for the central profile shape, the prior $L_{3}$ is activated. Thus, the central profile is forced to be nearly flat, while the mean density is set by the interferometer. Note that the upper part of the traditional reflectometry profile $\left(n_{e} \gtrsim 0.7 \cdot 10^{19}\right.$ $\mathrm{m}^{-3}$ ) is ignored, in accordance with the ambiguity analysis presented in Appendix A. To fully judge the match between the traditional reflectometry profile (green) and the Bayesian reconstructed profile (black), one would need to know the error in the traditional reconstruction. However, this information is not available; even so, the match seems to be reasonable, considering the local error of the Bayesian reconstruction (grey). The fine structure of the grey area (indicating the "local error level" of the reconstructed profile) reflects, in part, the structure of the prior $L_{3}$ ("knots" at $\rho=0,0.15,0.3,0.45,0.6$ ). We stress the importance of this result: at TJ-II, Thomson Scattering can deliver the core density profile only at a single time point; the present, new procedure allows reconstructing density profiles away from the TS time.

Fig. 8 shows the reconstruction based on the Thomson Scattering and interferometry data alone. Now, there are no edge data, so the prior $L_{2}$ (always activated) acts to keep the profile down at the edge. As a consequence, the profile drops to zero near the LCFS. The Thomson Scattering data are scaled up slightly (see Table II) so that the line-integrated density matches the interferometer value. Note the reduction of the "local error level" in the radial range $(0.65<\rho<0.8)$ where the density of data points is higher (due to the mapping from the laser beam chord to the magnetic geometry).

Fig. 9 shows the reconstruction based on interferometry, reflectometry and Thomson Scattering. Thus, a satisfactory reconstruction of the density profile is obtained by combining three diagnostics: interferometry sets the overall density value, establishing an appropriate value for the Thomson Scattering scaling factor $A_{T h}$, while Thomson Scattering provides the shape of the central density profile, and reflectometry provides the shape of the density profile in the edge.

Fig. 10 shows the reconstruction based on interferometry, reflectometry, Thomson Scat- 
tering, and the Helium beam. The He beam data provide independent confirmation of the preceding results; the relatively large size of the He beam error bars implies that its weight is not high, so the Bayesian profile is very similar to the one of Fig. 9 .

TABLE I. Shot $24729, t=1126.5 \mathrm{~ms}$, using a Bessel polynomial with $K$ terms, target line average density $\overline{n_{e}}=0.596\left(\right.$ units: $\left.10^{19} \mathrm{~m}^{-3}\right)$.

\begin{tabular}{|l|c|c|c|c|}
\hline Case & $K$ & $L$ & $\overline{n_{e}^{0}}$ & $A_{T h}$ \\
\hline Inter + refle & 4 & -374 & 0.592 & - \\
Inter + Thomson & 7 & -140 & 0.596 & 1.21 \\
Inter + refle + Thomson & 7 & -409 & 0.600 & 1.20 \\
Inter + refle + Thomson + He beam & 7 & -416 & 0.600 & 1.20 \\
\hline
\end{tabular}




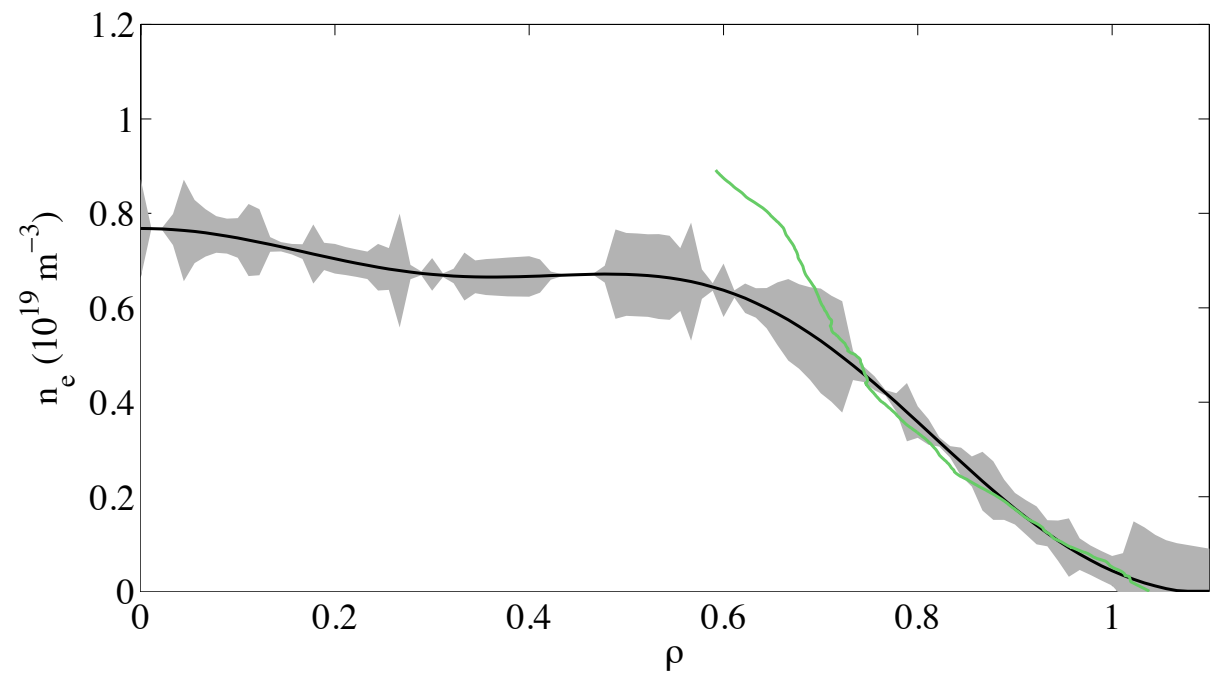

FIG. 7. Reconstructed profile using interferometry and reflectometry (see Table I). The traditional reflectometry profile (green) is followed closely, except for the high-density part (sensitive to profile ambiguity, as discussed in Appendix A). The central profile is approximately flat, in accordance with the prior $L_{3}$, while the mean density is set by the interferometer.

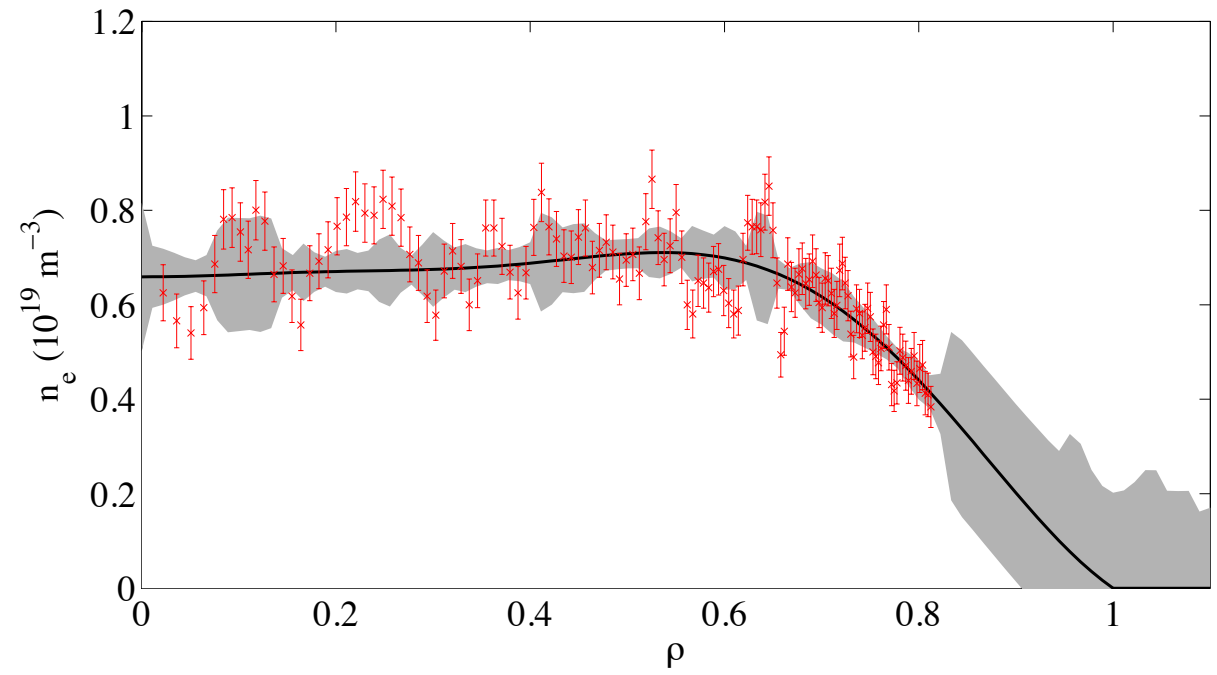

FIG. 8. Reconstructed profile using interferometry and Thomson scattering (see Table I). A good match with the Thomson profile is obtained, given the value of $K$ used (7). The density drops to zero near the LCFS, due to the prior $L_{2}$. 


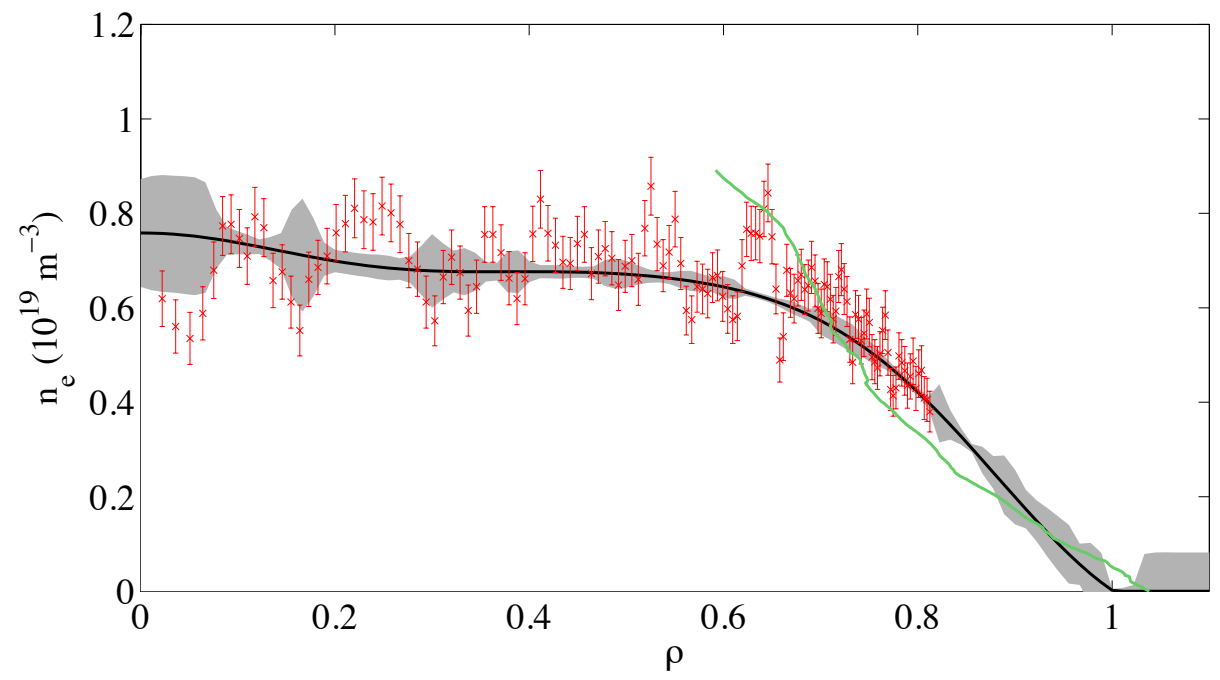

FIG. 9. Reconstructed profile using interferometry, reflectometry, and Thomson scattering (see Table I).

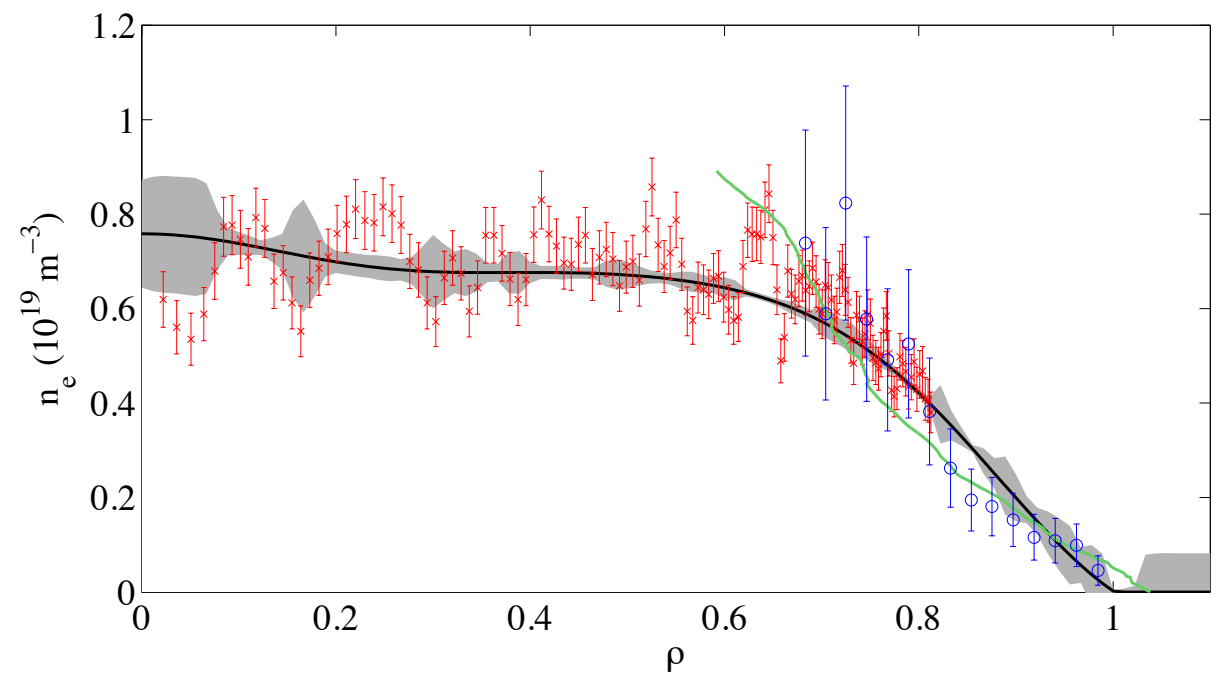

FIG. 10. Reconstructed profile using interferometry, reflectometry, Thomson scattering, and the Helium Beam (see Table I). 


\section{B. Biasing}

As another example, we show the reconstruction of the density profile in a set of similar discharges with electrode biasing (\# 16009 - 16015, configuration 100_44_64). The biasing electrode was inserted about $2 \mathrm{~cm}$ into the plasma $(\rho \simeq 0.9)$ and biased positively with respect to a poloidal limiter tangent to the Last Closed Flux Surface (LCFS), in the time interval $1100 \leq t \leq 1150 \mathrm{~ms}$. The biasing time interval and the response of the line integrated density (averaged over the 7 discharges in the series) are shown in Fig. 11. These

discharges have been analyzed in previous work ${ }^{29130}$. Significant long-range correlations (using toroidally separated Langmuir probes in the edge region) and bicoherence were detected, indicating the formation of an edge transport barrier associated with bias-induced shear flow.

Fig. 12 shows the reconstruction of the density profile based on the AM reflectometer (one profile scan every $2 \mathrm{~ms}$ ) and the interferometer, using the Bayesian method described above. To capture the detailed shape of the edge profile, we have set $K=15$, and to ensure a flat central density for $\rho<0.6$, we have set $n_{3}=10$ (in $L_{3}$ ). The radial position of the steepest gradient is $\rho \simeq 0.7$, unaffected by the application of the bias. At the very edge $(\rho \simeq 1)$, a steepening of the profile is visible. To view this in more detail, the density gradient at $\rho=1$ is shown in Fig. 13. The edge density gradient responds rapidly to the applied bias, more so than the mean particle density (especially at biasing switch-off), thus confirming the idea that electrode biasing generates an edge particle (electron) barrier. In relation to this, it is interesting that during the biasing time interval, the bicoherence, also assumed to be related to the formation of the edge transport barrier, was found to be concentrated in the radial range $0.9<\rho<1^{30}$. 


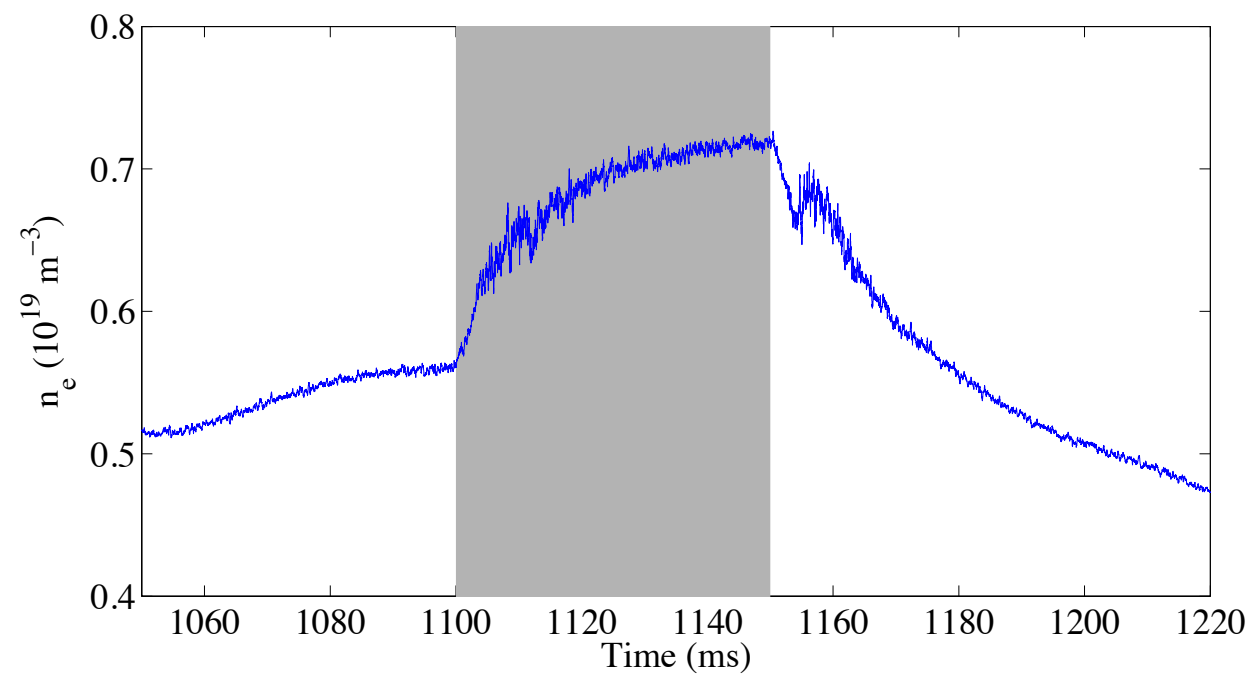

FIG. 11. Time trace of the line average density (interferometry), averaged over 7 similar discharges. The time window in which biasing is applied is indicated by a grey area.

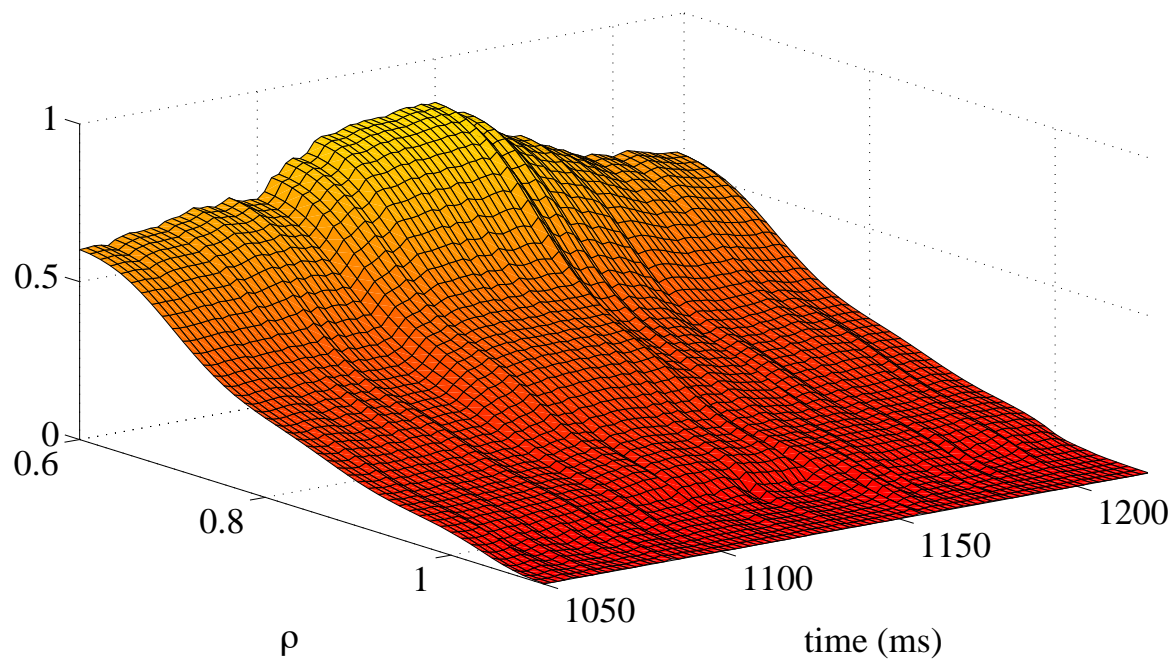

FIG. 12. Reconstruction of the density profile, based on reflectometry and interferometry, with $2 \mathrm{~ms}$ time resolution (the time of a frequency scan of the reflectometer), averaged over 7 similar discharges with electrode biasing $(1100<t<1150$, cf. Fig. 11). 


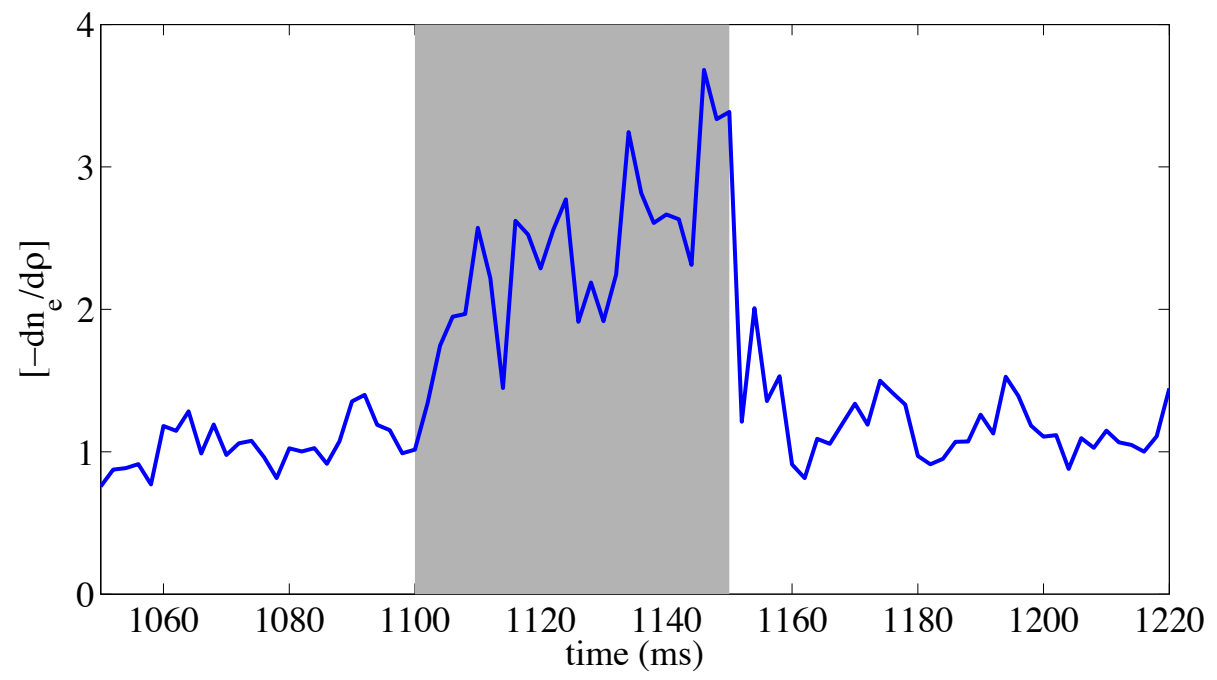

FIG. 13. Average edge density gradient, $-\left.\left\langle d n_{e} / d \rho\right\rangle\right|_{\rho=1}$, for 7 similar discharges. The time window in which biasing is applied is indicated by a grey area.. 

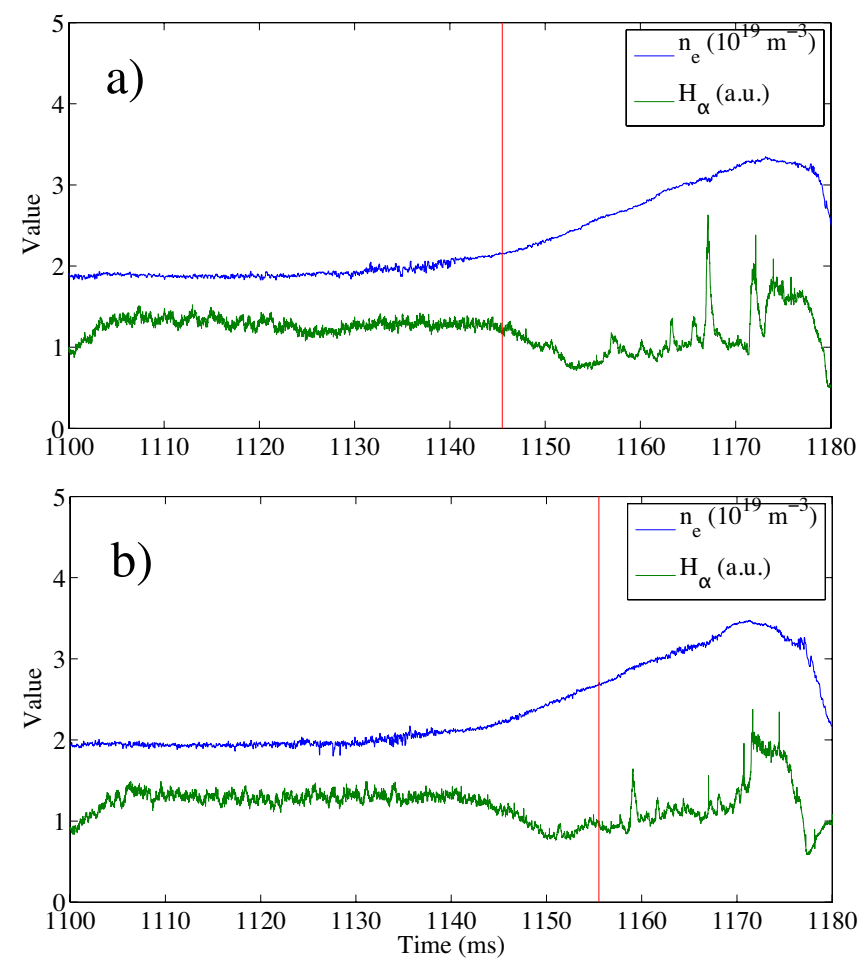

FIG. 14. Time traces of the line average density and a $H_{\alpha}$ monitor in two similar discharges with an L-H transition. The Thomson Scattering time is indicated by a vertical line: a) in discharge 23470 , this time is before the transition, and b) in discharge 23471, after.

\section{L- and H-mode plasma}

In this section, the technique described above is applied to two similar discharges (magnetic configuration: 100_32_60) with an "L-H" confinement transition. The general properties of such transitions at TJ-II are described in more detail elsewhere ${ }^{31}$. Discharge 23470 was in L-mode at the Thomson Scattering time $(t=1145.5)$, while discharge 23471 was in H-mode $(t=1155.5)$. Time traces of the line average density and a $H_{\alpha}$ monitor are shown in Fig. 14. The Thomson Scattering time is indicated by a vertical line.

The reconstruction is performed as described in the preceding sections, using all four diagnostics, at the TS times. The results are summarized in Table II and Fig. 15. There is general good agreement between the various diagnostics. The traditional reflectometer reconstruction is again affected by profile ambiguity for $n_{e} \gtrsim 0.6 \cdot 10^{19} \mathrm{~m}^{-3}$, as discussed in Appendix A. The reconstructed profiles clearly show the widening of the density profile when going from L to H; it appears a particle transport barrier forms at $\rho=0.74$. 
TABLE II. Shot 23470 (L) \& $23471(\mathrm{H})$.

\begin{tabular}{|c|c|c|c|c|c|}
\hline Case & $K$ & $L$ & $\overline{n_{e}}$ & $\overline{n_{e}^{0}}$ & $A_{T h}$ \\
\hline $23470(\mathrm{~L})$ & 12 & -507 & 2.154 & 2.154 & 1.005 \\
$23471(\mathrm{H})$ & 12 & -504 & 2.680 & 2.680 & 1.06 \\
\hline
\end{tabular}
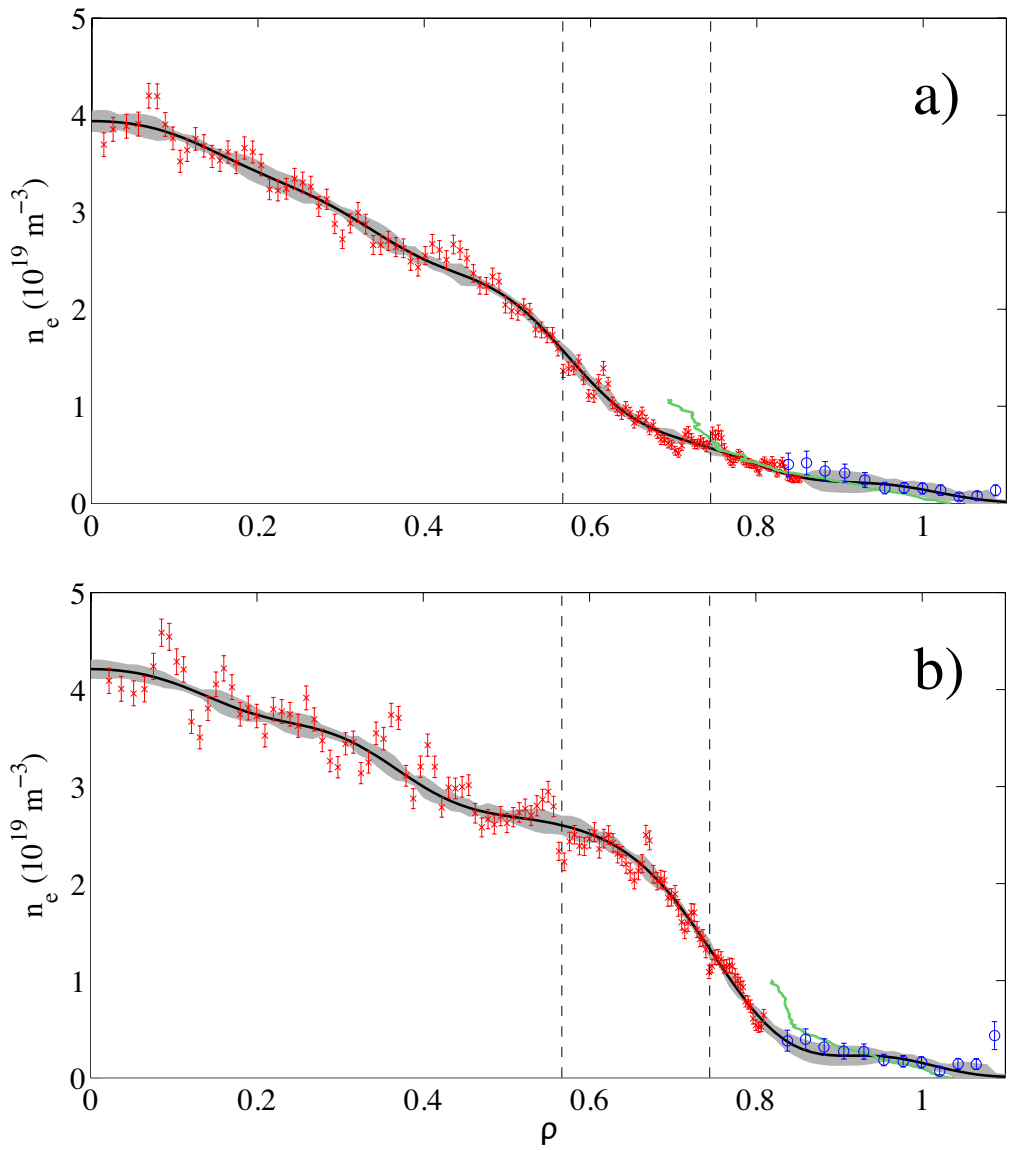

FIG. 15. a) L-mode; b) H-mode. Red crosses: scaled Thomson Scattering data points; green line: reflectometry profile (traditional reconstruction); blue circles: Helium beam data points; black line: reconstructed profile; grey area: local error estimate. The maximum gradient locations of the two profiles are indicated by vertical dashed lines to facilitate comparison. 


\section{CONCLUSIONS AND FUTURE WORK}

In this work, we have implemented an integrated data analysis procedure based on Bayesian inference at TJ-II for the first time.

We have developed new forward modelling simulations for the reflectometry and Helium beam diagnostics, including a detailed error analysis. We also presented a novel analysis of reflectometry profile reconstruction ambiguity, affecting traditional profile reconstruction methods (Appendix A), and showed how this ambiguity can be resolved using the Bayesian approach. The new analysis of the Helium beam emission is based on the use of Poisson statistics. The interferometry data are analyzed without recourse to the common assumption that the density is much smaller than the cut-off density.

We have demonstrated the successful reconstruction of profiles in both low-density and high-density plasmas, via maximization of the posterior probability. The Bayesian method allows defining priors that supplement the absence of certain diagnostics: e.g., in case information on the core profile is missing (no Thomson Scattering available), the central profile can be constrained by activating a prior to reduce the profile gradient in the central region, reflecting the a priori knowledge that density profiles are generally flat in the core of TJ-II (in ECRH plasmas). The latter procedure allows density profile reconstructions to be made at other times than the (single) TS time point. It should be noted that any uncertainty regarding the precise shape of the central density profile will lead to an additional uncertainty in the radial position of the external part of a profile that is reconstructed on the basis of reflectometry and interferometry alone. Additional information from the Helium beam and/or the Thomson scattering diagnostic, when available, serves to reduce this uncertainty.

We have shown how the gradual addition of diagnostics allows improving knowledge of the underlying physical density profile. We have applied the technique of marginalization to recover the error of the profile parameters, and have defined a more practical, local error estimate that can be used as a rough guide to the local precision of the profile.

We have applied the new profile reconstruction technique successfully to a typical ECRH plasma with moderate density, to a set of discharges with electrode biasing, and to a pair of NBI heated discharges with high density. The reconstructed time evolution of the density profile in the biasing discharges clearly shows the formation of an edge transport barrier in response to the biasing. The success of this analysis has prompted the study of such 
behaviour also in other (spontaneous) confinement transitions at TJ-II, providing enhanced insight into the mechanisms producing such transitions ${ }^{32}$. The NBI heated discharges were selected to provide an example of density profiles before and after an L-H transition. The formation of a particle transport barrier at $\rho=0.74$ could be identified with good precision. No low-order rational surfaces exist at this radial position. This fact is relevant for the study of the L-H transition mechanism at TJ-II and will be analyzed in more detail in the future.

TJ-II disposes of more profile-relevant diagnostics than the four discussed here. An overview is provided in Table III. The Lithium beam is discarded, as TJ-II currently employs Lithium for wall conditioning, thus blinding the diagnostic. A first extension of the present work would be to incorporate the Heavy Ion Beam Probe (HIBP), that provides local measurements of the density in the core region. Langmuir probes can provide data in the edge and Scrape-Off Layer.

In a second phase, the model can be enhanced to consider also the electron temperature $\left(T_{e}\right)$ profile. In this case, a full forward model for Thomson Scattering is available ${ }^{33}$ to model

the spectrum as obtained by the TS spectrograph. Once $T_{e}$ is modelled, forward modelling of the Helium beam line emission amplitude ratios is also possible. $T_{e}$ measurements from Electron Cyclotron Emission (ECE) can be considered, although this diagnostic is limited to low densities due to cutoff (therefore not suited for NBI heated discharges). The analysis can be enhanced further by adding information from the Soft X-Ray (SXR) diagnostic, possibly even allowing recovering some limited information on the effective charge profile, $Z_{\text {eff }}$.

Some of these diagnostics (SXR, bolometry) allow tomographic reconstructions to be made; so a future extension of the current work may also include, e.g., poloidal dependencies - this is however not immediate and must be considered a long-term goal.

\section{ACKNOWLEDGEMENTS}

The authors would like to thank the TJ-II Team for continued support and assistance. This research was sponsored in part by DGICYT (Dirección General de Investigaciones Científicas y Tecnológicas) of Spain under Project No. ENE2009-07247. The authors would like to acknowledge interesting discussions with J. Vega. 
TABLE III. Profile information delivered by diagnostics at TJ-II

\begin{tabular}{|l|c|c|c|c|}
\hline Diagnostic & $n_{e}$ & $T_{e}$ & $Z_{\text {eff }}$ & $\phi$ \\
\hline Interferometry & $\mathrm{X}$ & - & - & - \\
Reflectometry & $\mathrm{X}$ & - & - & - \\
Thomson Scattering & $\mathrm{X}$ & $\mathrm{X}$ & - & - \\
He beam & $\mathrm{X}$ & $\mathrm{X}$ & - & - \\
\hline Li beam & $\mathrm{X}$ & - & - & - \\
Langmuir probes & $\mathrm{X}$ & $\mathrm{X}$ & - & $\mathrm{X}$ \\
ECE & - & $\left(\right.$ only low $\left.n_{e}\right)$ & - & - \\
SXR & $\mathrm{X}$ & $\mathrm{X}$ & $\mathrm{X}$ & - \\
HIBP & $\mathrm{X}$ & - & - & $\mathrm{X}$ \\
\hline
\end{tabular}




\section{Appendix A: Reflectometry profile reconstruction}

The traditional reconstruction of density profiles from reflectometry data ${ }^{34}$ is of course subject to uncertainties due to measurement noise. However, in addition the reconstruction is subject to a level of ambiguity that is not related to measurement error but to the integration procedure of the profile reconstruction process, as discussed below. Note that this Appendix merely provides an analysis of this issue; none of the (approximate) equations of this Appendix are used for the actual profile reconstruction procedure in the main text of this paper.

The phase detected by the reflectometer is calculated by Eq. (14), repeated here for convenience:

$$
\phi(f)=\frac{4 \pi f}{c} \int_{r_{c}(f)}^{a} \eta(f, r) d r-\frac{\pi}{2}
$$

where $\eta$ is defined by Eq. (15). The critical radius $r_{c}(f)$ is the solution of $\eta(f, r)=0$, and the corresponding critical density is $(\omega=2 \pi f)$ :

$$
n_{c}(\omega)=n_{e}\left(r_{c}(\omega)\right)=\varepsilon_{0}\left(m_{e} \omega^{2} / e^{2}-B \omega / e\right),
$$

since only the smallest of the two possible solutions is relevant (the first cutoff). For sim-

plicity, the (mild) dependence of $\eta$ and $n_{c}$ on $r$ via $B(r)$ will be ignored here by taking $B$ constant; qualitatively, the discussion below is not affected by this dependence. Thus, one can express $\eta$ as a function of $f$ and $n_{e}$ (rather than $r$ ).

In view of this, it is convenient to rewrite the integral (A1) as an integral over the density, assuming that the density is a strictly monotonic function of $r$ for the relevant part of the density profile (the one probed by the reflectometer):

$$
\phi(f)=-\frac{4 \pi f}{c} \int_{0}^{n_{c}(f)} \eta\left(f, n_{e}\right)\left(\frac{d n_{e}}{d r}\right)^{-1} d n_{e}-\frac{\pi}{2}
$$

Written this way, the calculation only involves two parameters ( $f$ and $n_{e}$, considering $d n_{e} / d r$ to be a function of $\left.n_{e}\right)$ instead of three $\left(f, n_{e}\right.$, and $\left.r\right)$. It is seen that the measured phase is proportional to the mean inverse gradient, weighted by $\eta$. Note that knowledge of $d n_{e} / d r$ is sufficient to reconstruct the density profile in the relevant radial range, up to an offset.

Defining $G\left(n_{e}\right)=-\left(d n_{e} / d r\right)^{-1}$, and defining $\eta^{\prime}$ as

$$
\eta^{\prime}\left(f, n_{e}\right)=\left\{\begin{array}{cc}
\eta\left(f, n_{e}\right) & n_{e} \leq n_{c}(f) \\
0 & n_{e}>n_{c}(f)
\end{array}\right.
$$



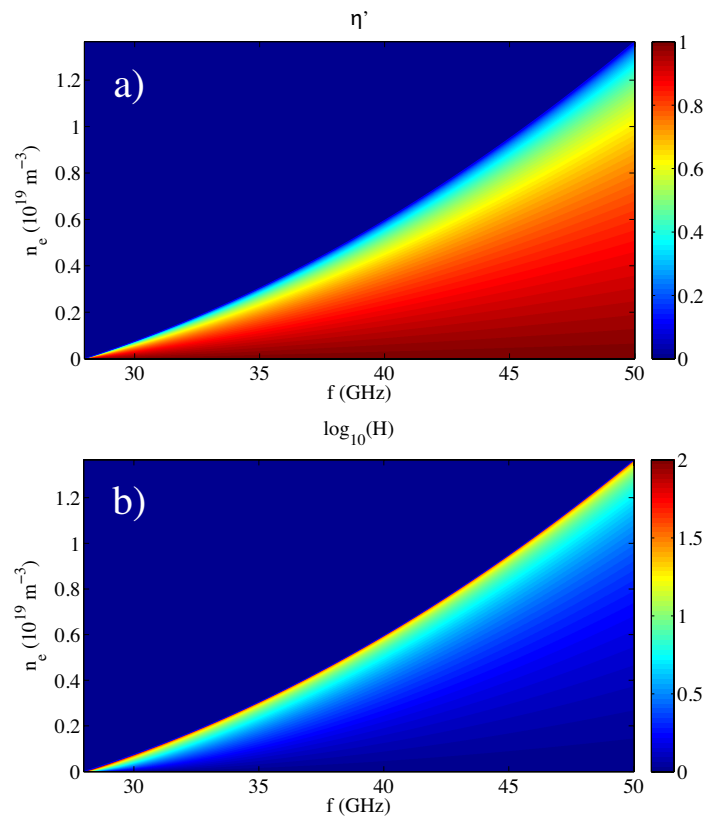

FIG. 16. a) The modified refractive index $\eta^{\prime}$ in the relevant parameter range for TJ-II. b) The integration kernel $H$.

one obtains

$$
\phi(f)=\frac{4 \pi}{c} \int_{0}^{n_{\max }} f \eta^{\prime}\left(f, n_{e}\right) G\left(n_{e}\right) d n_{e}-\frac{\pi}{2}
$$

where $n_{\max }=n_{c}\left(f_{\max }\right)$ (at least), and $f_{\max }$ is the maximum frequency of the frequency scan. For constant magnetic field $B(B=1 \mathrm{~T})$, the modified refractive index $\eta^{\prime}$ is easily evaluated, and is shown in Fig. 16 in the relevant parameter range.

Recall that the time delay $\tau(f)$ measured by the reflectometer is obtained from Eq. (13), resulting in

$$
\tau(f)=\frac{1}{2 \pi} \frac{d \phi(f)}{d f}=\frac{2}{c} \int_{0}^{n_{\max }} \frac{d\left(f \eta^{\prime}\left(n_{e}, f\right)\right)}{d f} G\left(n_{e}\right) d n_{e}
$$

The kernel of the integral is

$$
H\left(f, n_{e}\right)=\frac{d\left(f \eta^{\prime}\left(f, n_{e}\right)\right)}{d f}=\eta^{\prime}\left(f, n_{e}\right)+\frac{f}{2 \eta^{\prime}\left(f, n_{e}\right)} \frac{d \eta^{\prime 2}\left(f, n_{e}\right)}{d f}
$$

The second term in (A7) is strongly peaked at the critical density $(H$ is shown in Fig. 16 , right), and to first approximation one can set $H\left(f, n_{e}\right) \simeq g\left(f, n_{e}\right) \delta\left(n_{e}-n_{c}(f)\right)$, where $g$ is a slowly varying function. Effectively, the measured $\tau(f)$ is therefore almost proportional to $G\left(n_{c}(f)\right)$, i.e., the inverse density gradient at the critical density. This fact lies at the basis of the profile reconstruction procedure. 
Sampling the functions discretely, one can rewrite Eq.

$$
\vec{\tau}=\frac{2}{c} H^{\prime} \vec{G}
$$

where $\vec{\tau}$ is the vector of time delays, $\vec{G}$ the vector of the inverse density gradient values, and $H^{\prime}$ is computed by sampling $H$ discretely and multiplying by $\Delta n_{e}$ (the sampling interval of the density vector). Choosing the vectors of equal length so that $H^{\prime}$ is square, the matrix $H^{\prime}$ turns out to be rank-deficient (the rank being about $90 \%$ of its size). Therefore, strictly speaking, its inverse does not exist and the determination of $\vec{G}$ from $\vec{\tau}$ is subject to some degree of ambiguity: about 10\% of the inverse density gradient-eigenvectors are mapped to time delays $\overrightarrow{0}$ and cannot be recovered from the experimental data. The origin of the rank-deficiency is the non-linearity of the critical density line $n_{c}(f)$.

To show this graphically, we have decomposed $H^{\prime}=U S V^{T}$ using a standard Singular Value Decomposition. Here, $S$ is a diagonal matrix such that $S_{i i}=\lambda_{i}$ are the singular values. The columns of the $U$ and $V$ matrices form orthonormal basis vectors $\left(\vec{u}_{i}\right.$ and $\vec{v}_{i}$, respectively), the $i^{\text {th }}$ column vector corresponding to $\lambda_{i}$. To visualize the null space, we have summed the squared basis vectors $\vec{u}_{i}\left(f_{j}\right)=U_{j i}$ and $\vec{v}_{i}\left(\left(n_{e}\right)_{j}\right)=V_{j i}$ corresponding to small singular values $\left(\lambda_{i} \simeq 0\right.$ within computer precision). The result is shown in Fig. 17. Clearly the null space does not affect the reconstruction below $f=38.7 \mathrm{GHz}$ (or $n_{e}=0.66 \cdot 10^{19}$ $\mathrm{m}^{-3}$ ), but for higher values of the frequency and the density, the density of null space vector components is continuously increasing, so the difficulty of reconstructing the density profile unambiguously will increase gradually towards the high end of the range. The traditional reconstruction method does not take this ambiguity into account, so a profile is reconstructed that may be affected by such null vectors at high density values, not corresponding to any significant physical information.

Of course, this situation is slightly modified in the actual experimental situation due to the additional spatial variation of the magnetic field $B$ (which is, however, small in TJ-II). Also, the present analysis is 'ideal' in the sense that no signal loss or noise is considered; it merely provides an analysis of the profile reconstruction problem itself. We note that the issue of ambiguity is elegantly resolved using the Bayesian approach of this paper, as (a) only forward modelling is used, avoiding the problems associated with matrix inversion or similar profile reconstruction techniques, and (b) the reflectometry data are complemented with information from other diagnostics. 

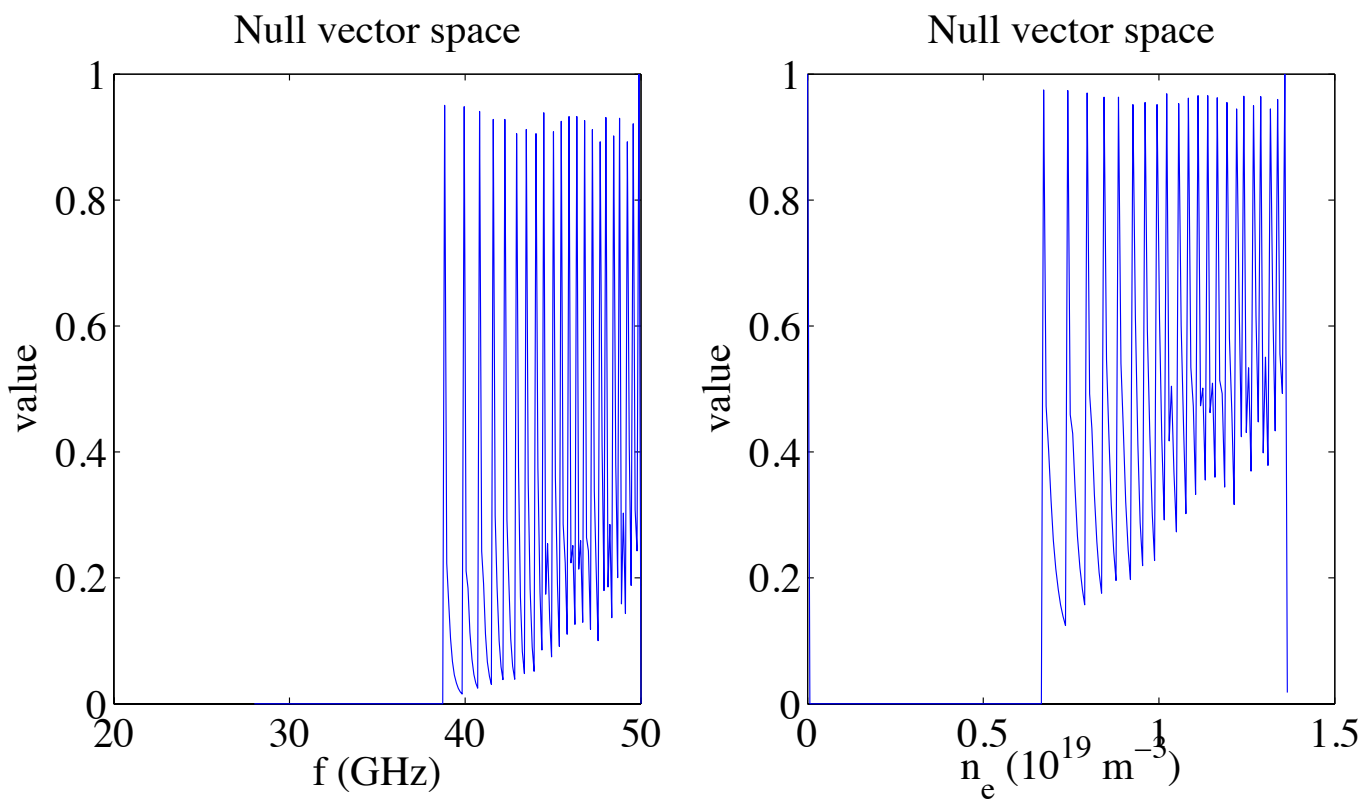

FIG. 17. Left: the null space of the $U$ basis vectors. Right: the null space of the $V$ basis vectors.

\section{Appendix B: The error of $\cos (\phi)$}

Assume one has a phase $\phi$, distributed uniformly over the interval $[0,2 \pi)$ (equivalent to a uniform distribution over the real line). It suffers a small, randomly distributed error $\delta \phi$, modeled as a Gaussian random number with mean 0 and standard deviation $\sigma$; this distribution will be denoted by $p_{G}(x)=\exp \left(-x^{2} / 2 \sigma^{2}\right) / \sqrt{2 \pi \sigma^{2}}$.

The error in $\cos \phi$ due to the error $\delta \phi$ is simply

$$
\delta \cos \phi=\cos (\phi+\delta \phi)-\cos \phi
$$

To first order in the errors, this is:

$$
\delta \cos \phi \simeq-\sin \phi \cdot \delta \phi
$$

Conservation of probability allows computing the distribution of $\delta \cos \phi$ :

$$
p_{G}(\delta \phi) d \delta \phi=p(\delta \cos \phi, \phi) d \delta \cos \phi
$$

where $p$ is the probability distribution of $\delta \cos \phi$, and all terms must be positive, so that

$$
p(\delta \cos \phi, \phi)=p_{G}(\delta \phi)\left|\frac{d \delta \phi}{d \delta \cos \phi}\right| \simeq p_{G}\left(\frac{\delta \cos \phi}{\sin \phi}\right) \frac{1}{|\sin \phi|}
$$


Of course, $\phi$ is an ignorable variable, so the mean distribution is obtained by averaging out $\phi$ :

$$
\overline{p(\delta \cos \phi)}=\frac{1}{2 \pi} \int_{0}^{2 \pi} p(\delta \cos \phi, \phi) d \phi
$$

with solution

$$
\overline{p(\delta \cos \phi)}=\pi^{-1} p_{G}\left(\frac{\delta \cos \phi}{\sqrt{2}}\right) K_{0}\left(\frac{\delta \cos \phi}{\sqrt{2}}\right)
$$

where $K_{0}$ is the modified Bessel function of the second kind. The width $w$ of this distribution (computed by evaluating the second moment, $w^{2}=\int x^{2} \overline{p(x)} d x$ ) is $w=\sigma / \sqrt{2}$.

Of course, the same result holds for the error in $\sin \phi$. 


\section{REFERENCES}

${ }^{1}$ D. Sivia, Data analysis: a Bayesian tutorial (Oxford University Press, New York, 1996).

${ }^{2}$ C. Alejaldre, J. Alonso, L. Almoguera, E. Ascasíbar, A. Baciero, R. Balbín, M. Blaumoser, J. Botija, B. Brañas, E. de la Cal, et al., Plasma Phys. Control. Fusion 41, A539 (1999).

${ }^{3}$ M. Hole, G. von Nessi, D. Pretty, J. Howard, B. Blackwell, J. Svensson, and L. Appel, Rev. Sci. Instrum. 81, 10E127 (2010).

${ }^{4}$ R. Fischer, A. Dinklage, and E. Pasch, Plasma Phys. Control. Fusion 45, 1095 (2003).

${ }^{5}$ R. Fischer, C. Wendland, A. Dinklage, S. Gori, V. Dose, and the W7-AS team, Plasma Physics and Controlled Fusion 44, 1501 (2002).

${ }^{6}$ A. Dinklage, R. Fischer, and J. Svensson, Fusion Sci. Technol. 46, 355 (2004).

${ }^{7}$ R. Fischer, C. Fuchs, B. Kurzan, W. Suttrop, E. Wolfrum, and ASDEX Upgrade Team, Fusion Sci. Technol. 58, 675 (2010).

${ }^{8}$ O. P. Ford, J. Svensson, A. Boboc, D. C. McDonald, and JET EFDA contributors, Plasma Physics and Controlled Fusion 51, 065004 (2009).

${ }^{9} \mathrm{P}$. Gregory, Bayesian logical data analysis for the physical sciences (Cambridge University Press, Cambridge, 2005).

${ }^{10}$ S. P. Hirshman, W. I. van Rij, and P. Merkel, Computer Physics Communications 43, 143 (1986).

${ }^{11}$ A. López-Fraguas, E. Ascasíbar, J. Romero, I. García-Cortés, J. Jiménez, A. LópezSánchez, J. Qin, M. Otte, and the TJ-II Team, Proc. $13^{\text {th }}$ International Stellarator Workshop, Canberra, Australia (2002) pp. P1-6 (2002).

${ }^{12}$ E. Ascasíbar, J. Qin, and A. López-Fraguas, J. Plasma Fusion Res. SERIES 1, 183 (1998).

${ }^{13}$ V. Tribaldos, Phys. Plasmas 8, 1229 (2001).

${ }^{14}$ J. Romero, D. López-Bruna, A. López-Fraguas, E. Ascasíbar, and the TJ-II Team, Nucl. Fusion 43, 387 (2003).

${ }^{15}$ D. López-Bruna, F. Castejón, T. Estrada, J. Romero, J. Jiménez, E. Ascasíbar, and the TJ-II Team, Nucl. Fusion 44, 645 (2004).

${ }^{16} \mathrm{~V}$. Tribaldos, J. Jiménez, J. Guasp, and B. van Milligen, Plasma Phys. Control. Fusion 40, 2113 (1998).

${ }^{17}$ R. Fischer, E. Wolfrum, J. Schweinzer, and the ASDEX Upgrade Team, Plasma Phys. Control. Fusion 50, 085009 (2008). 
${ }^{18}$ I. Hutchinson, Principles of Plasma Diagnostics (Cambridge University Press, New York, 2002).

${ }^{19}$ B. van Milligen, T. Estrada, R. Jiménez-Gómez, A. Melnikov, C. Hidalgo, J. Fontdecaba, L. Krupnik, L. Eliseev, S. Perfilov, and the TJ-II Team, Nucl. Fusion 51, 013005 (2011).

${ }^{20}$ T. Estrada, J. Sánchez, B. van Milligen, L. Cupido, A. Silva, M. Manso, and V. Zhuravlev, Plasma Phys. Control. Fusion 43, 1535 (2001).

${ }^{21}$ J. Sánchez, B. Brañas, T. Estrada, E. de la Luna, and V. Zhuravlev, Rev. Sci. Instrum. 63, 4654 (1992).

${ }^{22}$ C. Laviron, A. Donné, M. Manso, and J. Sánchez, Plasma Phys. Control. Fusion 38, 905 (1996).

${ }^{23}$ C. Barth, F. Pijper, H. van der Meiden, J. Herranz, and I. Pastor, Rev. Sci. Instrum. 70, $763(1999)$.

${ }^{24}$ J. Vega, C. Crémy, E. Sánchez, A. Portas, J. Fábregas, and R. Herrera, Fusion Eng. Des. 48, 69 (2000).

${ }^{25}$ B. Brañas, D. Tafalla, F. Tabarés, and P. Ortiz, Rev. Sci. Instrum. 72, 602 (2001).

${ }^{26}$ A. Hidalgo, D. Tafalla, B. Brañas, and F. Tabarés, Rev. Sci. Instrum. 75, 3478 (2004).

${ }^{27}$ NAG Fortran Library Manual, Mark 19 (The Numerical Algorithms Group Ltd., Oxford, 1999).

${ }^{28}$ W. Press, S. Teukolsky, W. Vetterling, and B. Flannery, Numerical Recipes in FORTRAN (Cambridge University Press, 1992), 2nd ed.

${ }^{29}$ M. Pedrosa, C. Silva, C. Hidalgo, B. Carreras, R. Orozco, D. Carralero, and the TJ-II Team, Phys. Rev. Lett. 100, 215003 (2008).

${ }^{30}$ B. van Milligen, T. Kalhoff, M. Pedrosa, and C. Hidalgo, Nucl. Fusion 48, 115003 (2008).

${ }^{31}$ J. Sánchez, M. Acedo, A. Alonso, J. Alonso, P. Alvarez, E. Ascasíbar, A. Baciero, R. Balbín, L. Barrera, E. Blanco, et al., Nuclear Fusion 49, 104018 (2009).

${ }^{32}$ B. van Milligen, M. Pedrosa, C. Hidalgo, B. Carreras, T. Estrada, J. Alonso, J. de Pablos, A. Melnikov, L. Krupnik, L. Eliseev, et al., Nucl. Fusion Submitted (2011).

${ }^{33}$ B. van Milligen, I. Classen, and C. Barth, Rev. Sci. Instrum. 74, 3998 (2003).

${ }^{34}$ H. Bottolier-Curtet and G. Ichtchenko, Rev. Sci. Instrum. 58, 539 (1987). 